\title{
Approximation of Common Solutions to System of Mixed Equilibrium Problems, Variational Inequality Problem, and Strict Pseudo-Contractive Mappings
}

\author{
Poom Kumam ${ }^{1,2}$ and Chaichana Jaiboon ${ }^{2,3}$ \\ ${ }^{1}$ Department of Mathematics, Faculty of Science, King Mongkut's University of \\ Technology Thonburi (KMUTT), Bangkok 10140, Thailand \\ ${ }^{2}$ Centre of Excellence in Mathematics, CHE, Si Ayuthaya Road, Bangkok 10400, Thailand \\ ${ }^{3}$ Department of Mathematics, Faculty of Liberal Arts, Rajamangala University of \\ Technology Rattanakosin (RMUTR), Bangkok 10100, Thailand
}

Correspondence should be addressed to Chaichana Jaiboon, chaichana.j@rmutr.ac.th

Received 3 October 2010; Accepted 5 March 2011

Academic Editor: Jong Kim

Copyright (c) 2011 P. Kumam and C. Jaiboon. This is an open access article distributed under the Creative Commons Attribution License, which permits unrestricted use, distribution, and reproduction in any medium, provided the original work is properly cited.

We introduce an iterative algorithm for finding a common element of the set of fixed points of strict pseudocontractions mapping, the set of common solutions of a system of two mixed equilibrium problems and the set of common solutions of the variational inequalities with inverse strongly monotone mappings. Strong convergence theorems are established in the framework of Hilbert spaces. Finally, we apply our results for solving convex feasibility problems in Hilbert spaces. Our results improve and extend the corresponding results announced by many others recently.

\section{Introduction}

Throughout this paper, we denote by $N$ and $R$ the sets of positive integers and real numbers, respectively. Let $H$ be a real Hilbert space with inner product $\langle\cdot, \cdot\rangle$ and norm $\|\cdot\|$, and let $E$ be a nonempty closed convex subset of $H$. We denote weak convergence and strong convergence by notations $\rightarrow$ and $\rightarrow$, respectively. Recall that a mapping $f: E \rightarrow E$ is an $\alpha$-contraction on $E$ if there exists a constant $\alpha \in(0,1)$ such that $\|f(x)-f(y)\| \leq \alpha\|x-y\|$ for all $x, y \in E$. Let $S: E \rightarrow E$ be a mapping. In the sequel, we will use $F(S)$ to denote the set of fixed points of $S$; that is, $F(S)=\{x \in E: S x=x\}$. In addition, let a mapping $S: E \rightarrow E$ be called nonexpansive, if $\|S x-S y\| \leq\|x-y\|$, for all $x, y \in E$. It is well known that if $E \subset H$ is nonempty, bounded, closed, and convex and $S$ is a nonexpansive self-mapping on $E$, then $F(S)$ is nonempty; see, 
for example, [1]. Recall that a mapping $S: E \rightarrow E$ is called strictly pseudo-contraction if there exists a constant $k \in[0,1)$ such that

$$
\|S x-S y\|^{2} \leq\|x-y\|^{2}+k\|(I-S) x-(I-S) y\|^{2}, \quad \forall x, y \in E,
$$

where $I$ denotes the identity operator on $E$. Note that if $k=0$, then $S$ is a nonexpansive mapping. The class of strict pseudo-contractions is one of the most important classes of mappings among nonlinear mappings. Within the past several decades, many authors have been devoted to the studies on the existence and convergence of fixed points for strict pseudocontractions. In 1967, Browder and Petryshyn [2] introduced a convex combination method to study strict pseudo-contractions in Hilbert spaces. On the other hand, Marino and $\mathrm{Xu}$ [3] and Zhou [4] developed some iterative scheme for finding a fixed point of a strict pseudocontraction mapping. More precisely, take $k \in(0,1)$ and define a mapping $S_{k}$ by

$$
S_{k} x=k x+(1-k) S x, \quad \forall x \in E,
$$

where $S$ is a strict pseudo-contraction. Under appropriate restrictions on $k$, it is proved that the mapping $S_{k}$ is nonexpansive. Therefore, the techniques of studying nonexpansive mappings can be applied to study more general strict pseudo-contractions.

Let $\varphi: E \rightarrow R \cup\{+\infty\}$ be a proper extended real-valued function and let $\phi$ be a bifunction of $E \times E$ into $\mathcal{R}$ such that $E \cap \operatorname{dom} \varphi \neq \emptyset$, where $\mathcal{R}$ is the set of real numbers and $\operatorname{dom} \varphi=\{x \in E: \varphi(x)<+\infty\}$. Ceng and Yao [5] considered the following mixed equilibrium problems for finding $x \in E$ such that

$$
\phi(x, y)+\varphi(y)-\varphi(x) \geq 0, \quad \forall y \in E
$$

The set of solutions of $(1.3)$ is denoted by $\operatorname{MEP}(\phi, \varphi)$, that is,

$$
\operatorname{MEP}(\phi, \varphi)=\{x \in E: \phi(x, y)+\varphi(y)-\varphi(x) \geq 0, \forall y \in E\}
$$

We see that $x$ is a solution of a problem (1.3) that implies that $x \in \operatorname{dom} \varphi=\{x \in E$ : $\varphi(x)<+\infty\}$.

Special Examples

(1) If $\varphi=0$, then the mixed equilibrium problem (1.3) becomes to be the equilibrium problem which is to find $x \in E$ such that

$$
\phi(x, y) \geq 0, \quad \forall y \in E
$$

The set of solutions of (1.5) is denoted by $\operatorname{EP}(\phi)$.

(2) If $\varphi=0$ and $\phi(x, y)=\langle B x, y-x\rangle$ for all $x, y \in E$, where $B: E \rightarrow H$ is a nonlinear mapping, then problem (1.5) becomes to be the variational inequality problems which is to find $x \in E$ such that

$$
\langle B x, y-x\rangle \geq 0, \quad \forall y \in E
$$


The set of solutions of (1.6) is denoted by $\operatorname{VI}(E, B)$. The variational inequality has been extensively studied in the literature. See, for example, [6-8] and the references therein.

The mixed equilibrium problems include fixed point problems, variational inequality problems, optimization problems, Nash equilibrium problems, and the equilibrium problem as special cases. Numerous problems in physics, optimization, and economics reduce to find a solution of (1.3). Some authors have proposed some useful methods for solving the $\operatorname{MEP}(\phi, \varphi)$ and $\operatorname{EP}(\phi)$; see, for instance [5, 9-27]. In 1997, Combettes and Hirstoaga [10] introduced an iterative scheme of finding the best approximation to initial data when $\operatorname{EP}(\phi)$ is nonempty and proved a strong convergence theorem. Next, we recall some definitions.

Definition 1.1. Let $B: E \rightarrow H$ be nonlinear mappings. Then $B$ is called

(1) monotone if

$$
\langle B x-B y, x-y\rangle \geq 0, \quad \forall x, y \in E,
$$

(2) $\rho$-strongly monotone if there exists a constant $\rho>0$ such that

$$
\langle B x-B y, x-y\rangle \geq \rho\|x-y\|^{2}, \quad \forall x, y \in E,
$$

(3) $\eta$-Lipschitz continuous if there exists a constant $\eta>0$ such that

$$
\|B x-B y\| \leq \eta\|x-y\|, \quad \forall x, y \in E
$$

(4) $\beta$-inverse strongly monotone if there exists a constant $\beta>0$ such that

$$
\langle B x-B y, x-y\rangle \geq \beta\|B x-B y\|^{2}, \quad \forall x, y \in E
$$

Remark 1.2. It is obvious that any $\beta$-inverse strongly monotone mappings $B$ is monotone and $(1 / \beta)$-Lipschitz continuous.

(5) A set-valued mapping $T: H \rightarrow 2^{H}$ is called a monotone if, for all $x, y \in H, f \in T x$ and $g \in T y$ imply $\langle x-y, f-g\rangle \geq 0$.

(6) A monotone mapping $T: H \rightarrow 2^{H}$ is a maximal if the graph of $G(T)$ of $T$ is not properly contained in the graph of any other monotone mapping. It is known that a monotone mapping $T$ is maximal if and only if for $(x, f) \in H \times H,\langle x-y, f-g\rangle \geq 0$ for every $(y, g) \in G(T)$ implies $f \in T x$.

Let $B$ be a monotone map of $E$ into $H, \eta$-Lipschitz continuous mapping and let $N_{E} \vartheta$ be the normal cone to $E$ when $\vartheta \in E$, that is,

$$
N_{E} \vartheta=\{w \in H:\langle u-\vartheta, w\rangle \geq 0, \forall u \in E\}
$$


and define a mapping $T$ on $E$ by

$$
T \vartheta= \begin{cases}B \vartheta+N_{E} \vartheta, & \vartheta \in E, \\ \emptyset, & \vartheta \notin E .\end{cases}
$$

Then $T$ is the maximal monotone and $0 \in T \vartheta$ if and only if $\vartheta \in \operatorname{VI}(E, B)$; see [28].

For finding a common element of the set of fixed points of a nonexpansive mapping and the set of solution of variational inequalities for $\beta$-inverse strongly monotone, Takahashi and Toyoda [29] first introduced the following iterative scheme:

$$
\begin{gathered}
x_{0} \in E \quad \text { chosen arbitrary, } \\
x_{n+1}=\alpha_{n} x_{n}+\left(1-\alpha_{n}\right) S P_{E}\left(x_{n}-\lambda_{n} B x_{n}\right), \quad \forall n \geq 0,
\end{gathered}
$$

where $B$ is an $\beta$-inverse strongly monotone, $\left\{\alpha_{n}\right\}$ is a sequence in $(0,1)$, and $\left\{\lambda_{n}\right\}$ is a sequence in $(0,2 \beta)$. They showed that if $F(S) \cap \operatorname{VI}(E, B)$ is nonempty, then the sequence $\left\{x_{n}\right\}$ generated by (1.13) converges weakly to some $q \in F(S) \cap \operatorname{VI}(E, B)$.

Further, Y. Yao and J.-C. Yao [30] introduced the following iterative scheme:

$$
\begin{gathered}
x_{1}=x \in E \quad \text { chosen arbitrary, } \\
y_{n}=P_{E}\left(x_{n}-\lambda_{n} B x_{n}\right), \\
x_{n+1}=\alpha_{n} x+\beta_{n} x_{n}+\gamma_{n} S P_{E}\left(y_{n}-\lambda_{n} B y_{n}\right), \quad \forall n \geq 1,
\end{gathered}
$$

where $B$ is an $\beta$-inverse strongly monotone, $\left\{\alpha_{n}\right\},\left\{\beta_{n}\right\},\left\{\gamma_{n}\right\}$ are three sequences in $[0,1]$, and $\left\{\lambda_{n}\right\}$ is a sequence in $(0,2 \beta)$. They showed that if $F(S) \cap \operatorname{VI}(E, B)$ is nonempty, then the sequence $\left\{x_{n}\right\}$ generated by (1.14) converges strongly to some $q \in F(S) \cap \operatorname{VI}(E, B)$.

A map $A: H \rightarrow H$ is said to be strongly positive if there exists a constant $\bar{\gamma}>0$ such that

$$
\langle A x, x\rangle \geq \bar{\gamma}\|x\|^{2}, \quad \forall x \in H .
$$

A typical problem is to minimize a quadratic function over the set of the fixed points of some nonexpansive mapping on a real Hilbert space $H$ :

$$
\min _{x \in E} \frac{1}{2}\langle A x, x\rangle-\langle x, b\rangle \text {, }
$$

where $A$ is some linear, $E$ is the fixed point set of a nonexpansive mapping $S$ on $H$ and $b$ is a point in $H$. Let $A$ be a strongly positive linear bounded map on $H$ with coefficient $\gamma$. In 2006, Marino and $\mathrm{Xu}$ [31] studied the following general iterative method:

$$
x_{n+1}=\epsilon_{n} \gamma f\left(x_{n}\right)+\left(1-\epsilon_{n} A\right) S x_{n} .
$$


They proved that if the sequence $\epsilon_{n}$ of parameters appropriate conditions, then the sequence $x_{n}$ generated by (1.17) converges strongly to $q=P_{F(S)}(I-A+\gamma f)(q)$. Recently, Plubtieng and Punpaeng [32] proposed the following iterative algorithm:

$$
\begin{gathered}
\phi\left(u_{n}, y\right)+\frac{1}{r_{n}}\left\langle y-u_{n}, u_{n}-x_{n}\right\rangle \geq 0, \quad \forall y \in H \\
x_{n+1}=\epsilon_{n} \gamma f\left(x_{n}\right)+\left(I-\epsilon_{n} A\right) S u_{n} .
\end{gathered}
$$

They proved that if the sequences $\left\{\epsilon_{n}\right\}$ and $\left\{r_{n}\right\}$ of parameters satisfy appropriate condition, then both sequences $\left\{x_{n}\right\}$ and $\left\{u_{n}\right\}$ converge to the unique solution $q$ of the variational inequality

$$
\langle(A-\gamma f) q, x-q\rangle \geq 0, \quad \forall x \in F(S) \cap \mathrm{EP}(\phi)
$$

which is the optimality condition for the minimization problem

$$
\min _{x \in F(S) \cap \mathrm{EP}(\phi)} \frac{1}{2}\langle A x, x\rangle-h(x)
$$

where $h$ is a potential function for $\gamma f$ (i.e., $h^{\prime}(x)=\gamma f(x)$ for $x \in H$ ).

On the other hand, for finding a common element of the set of fixed points of a $k$ strict pseudo-contraction mapping and the set of solutions of an equilibrium problem in a real Hilbert space, Liu [33] introduced the following iterative scheme:

$$
\begin{gathered}
\phi\left(u_{n}, y\right)+\frac{1}{r_{n}}\left\langle y-u_{n}, u_{n}-x_{n}\right\rangle \geq 0, \quad \forall y \in E, \\
y_{n}=\beta_{n} u_{n}+\left(1-\beta_{n}\right) S u_{n}, \\
x_{n+1}=\epsilon_{n} \gamma f\left(x_{n}\right)+\left(I-\epsilon_{n} A\right) u_{n}, \quad \forall n \geq 1,
\end{gathered}
$$

where $S$ is a $k$-strict pseudo-contraction mapping and $\left\{\epsilon_{n}\right\},\left\{\beta_{n}\right\}$ are sequences in $[0,1]$. They proved that under certain appropriate conditions over $\left\{\epsilon_{n}\right\},\left\{\beta_{n}\right\}$, and $\left\{r_{n}\right\}$, the sequences $\left\{x_{n}\right\}$ and $\left\{u_{n}\right\}$ converge strongly to some $q \in F(S) \cap \operatorname{EP}(\phi)$, which solves some variational inequality problems.

In 2008, Ceng and Yao [5] introduced an iterative scheme for finding a common fixed point of a finite family of nonexpansive mappings and the set of solutions of a problem (1.3) in Hilbert spaces and obtained the strong convergence theorem which used the following condition:

(H) $K: E \rightarrow \mathcal{R}$ is $\eta$-strongly convex with constant $\sigma>0$ and its derivative $K^{\prime}$ is sequentially continuous from weak topology to strong topology. We note that the condition (H) for the function $K: E \rightarrow R$ is a very strong condition. We also note that the condition (H) does not cover the case $K(x)=\|x\|^{2} / 2$ and $\eta(x, y)=x-y$ for each $(x, y) \in E \times E$. Very recently, R. Wangkeeree and R. Wangkeeree [34] introduced a general iterative method for finding a common element of the set of solutions of the mixed equilibrium problems, the set of fixed point of a $k$-strict pseudo-contraction mapping, and the set of solutions of 
the variational inequality for an inverse strongly monotone mapping in Hilbert spaces. They obtained a strong convergence theorem except the condition $(\mathrm{H})$ for the sequences generated by these processes.

In 2009, Qin et al. [35] introduced a general iterative scheme for finding a common element of the set of common solution of generalized equilibrium problems, the set of a common fixed point of a family of infinite nonexpansive mappings in Hilbert spaces. Let $\left\{x_{n}\right\}$ be the sequence generated iterative by the following algorithm:

$$
\begin{gathered}
x_{1} \in E, \quad u_{n} \in E, \quad v_{n} \in E, \\
\phi_{1}\left(u_{n}, u\right)+\left\langle C x_{n}, u-u_{n}\right\rangle+\frac{1}{r}\left\langle u-u_{n}, u_{n}-x_{n}\right\rangle \geq 0, \quad \forall u \in E, \\
\phi_{2}\left(v_{n}, v\right)+\left\langle B x_{n}, v-v_{n}\right\rangle+\frac{1}{S}\left\langle v-v_{n}, v_{n}-x_{n}\right\rangle \geq 0, \quad \forall v \in E, \\
y_{n}=\delta_{n} u_{n}+\left(1-\delta_{n}\right) v_{n}, \\
x_{n+1}=\epsilon_{n} f\left(x_{n}\right)+\beta_{n} x_{n}+\gamma_{n} W_{n} y_{n}, \quad \forall n \geq 1 .
\end{gathered}
$$

They proved that under certain appropriate conditions imposed on $\left\{\epsilon_{n}\right\},\left\{\beta_{n}\right\},\left\{\gamma_{n}\right\}$ and $\left\{\delta_{n}\right\}$, the sequence $\left\{x_{n}\right\}$ generated by (1.22) converges strongly to $q \in \cap_{n=1}^{\infty} F\left(T_{n}\right) \cap \operatorname{EP}\left(\phi_{1}, C\right) \cap$ $\operatorname{EP}\left(\phi_{2}, B\right)$, where $q=P_{\cap_{n=1}^{\infty}} F\left(T_{n}\right) \cap \operatorname{EP}\left(\phi_{1}, C\right) \cap \operatorname{EP}\left(\phi_{2}, B\right) f(q)$.

In the present paper, motivated and inspired by Qin et al. [35], Plubtieng and Punpaeng [32], Peng and Yao [17], R. Wangkeeree and R. Wangkeeree [34], and Y. Yao and J.-C. Yao [30], we introduce a new approximation iterative scheme for finding a common element of the set of fixed points of strict pseudo-contractions, the set of common solutions of the system of a mixed equilibrium problem, and the set of common solutions of the variational inequalities with inverse strongly monotone mappings in Hilbert spaces. We obtain a strong convergence theorem for the sequences generated by these processes under some parameter controlling conditions. Moreover, we apply our results for solving convex feasibility problems in Hilbert spaces. The results in this paper extend and improve some well-known results in $[17,30,32,34,35]$.

\section{Preliminaries}

Let $H$ be a real Hilbert space and $E$ be a closed convex subset of $H$. In a real Hilbert space $H$, it is well known that

$$
\|\lambda x+(1-\lambda) y\|^{2}=\lambda\|x\|^{2}+(1-\lambda)\|y\|^{2}-\lambda(1-\lambda)\|x-y\|^{2}
$$

for all $x, y \in H$ and $\lambda \in[0,1]$.

For any $x \in H$, there exists a unique nearest point in $E$, denoted by $P_{E} x$, such that

$$
\left\|x-P_{E} x\right\| \leq\|x-y\|, \quad \forall y \in E
$$

The mapping $P_{E}$ is called the metric projection of $H$ onto $E$. 
It is well known that $P_{E}$ is a firmly nonexpansive mapping of $H$ onto $E$, that is,

$$
\left\langle x-y, P_{E} x-P_{E} y\right\rangle \geq\left\|P_{E} x-P_{E} y\right\|^{2}, \quad \forall x, y \in H
$$

Further, for any $x \in H$ and $z \in E, z=P_{E} x$ if and only if $\langle x-z, z-y\rangle \geq 0$, for all $y \in E$. Moreover, $P_{E} x$ is characterized by the following properties: $P_{E} x \in E$ and

$$
\begin{gathered}
\left\langle x-P_{E} x, y-P_{E} x\right\rangle \leq 0, \\
\|x-y\|^{2} \geq\left\|x-P_{E} x\right\|^{2}+\left\|y-P_{E} x\right\|^{2}
\end{gathered}
$$

for all $x \in H, y \in E$.

It is easy to see that the following is true:

$$
u \in \mathrm{VI}(E, B) \Longleftrightarrow u=P_{E}(u-\lambda B u), \quad \curlywedge>0 .
$$

The following lemmas will be useful for proving the convergence result of this paper.

Lemma 2.1 (see [36]). Let $(E,\langle\cdot, \cdot\rangle)$ be an inner product space. Then, for all $x, y, z \in E$ and $\alpha, \beta, \gamma \in$ $[0,1]$ with $\alpha+\beta+\gamma=1$, one has

$$
\|\alpha x+\beta y+\gamma z\|^{2}=\alpha\|x\|^{2}+\beta\|y\|^{2}+\gamma\|z\|^{2}-\alpha \beta\|x-y\|^{2}-\alpha \gamma\|x-z\|^{2}-\beta \gamma\|y-z\|^{2} .
$$

Lemma 2.2 (see [31]). Assume that $A$ is a strongly positive linear bounded operator on $H$ with coefficient $\bar{\gamma}>0$ and $0<\rho \leq\|A\|^{-1}$. Then $\|I-\rho A\| \leq 1-\rho \bar{\gamma}$.

Lemma 2.3 (see [4]). Let $E$ be a nonempty closed convex subset of a real Hilbert space $H$ and let $S: E \rightarrow E$ be a $k$-strict pseudo-contraction with a fixed point. Then $F(S)$ is closed and convex. Define $S_{k}: E \rightarrow E$ by $S_{k}=k x+(1-k) S x$ for each $x \in E$. Then $S_{k}$ is nonexpansive such that $F\left(S_{k}\right)=F(S)$.

Lemma 2.4 (see [37]). Let $X$ be a uniformly convex Banach spaces, E be a nonempty closed convex subset of $X$ and $S: E \rightarrow E$ be a nonexpansive mapping. Then $I-S$ is demi-closed at zero.

Lemma 2.5 (see [38]). Let E be a nonempty closed convex subset of strictly convex Banach space X. Let $\left\{T_{n}: n \in \mathbb{N}\right\}$ be a sequence of nonexpansive mappings on $E$. Suppose $\cap_{n=1}^{\infty} F\left(T_{n}\right)$ is nonempty. Let $\delta_{n}$ be a sequence of positive numbers with $\sum_{n=1}^{\infty} \delta_{n}=1$. Then a mapping $S$ on $E$ can be defined by

$$
S x=\sum_{n=1}^{\infty} \delta_{n} T_{n} x
$$

for $x \in E$ is well defined, nonexpansive and $F(S)=\cap_{n=1}^{\infty} F\left(T_{n}\right)$ holds. 
In order to solve the mixed equilibrium problem, the following assumptions are given for the bifunction $\phi, \varphi$ and the set $E$ :

(A1) $\phi(x, x)=0$ for all $x \in E$;

(A2) $\phi$ is monotone, that is, $\phi(x, y)+\phi(y, x) \leq 0$ for all $x, y \in E$;

(A3) for each $x, y, z \in E, \lim _{t \rightarrow 0} \phi(t z+(1-t) x, y) \leq \phi(x, y)$;

(A4) for each $x \in E, y \mapsto \phi(x, y)$ is convex and lower semicontinuous;

(A5) for each $y \in E, x \mapsto \phi(x, y)$ is weakly upper semicontinuous;

(B1) for each $x \in H$ and $r>0$, there exist abounded subset $D_{x} \subseteq E$ and $y_{x} \in E$ such that for any $z \in E \backslash D_{x}$,

$$
\phi\left(z, y_{x}\right)+\varphi\left(y_{x}\right)-\varphi(z)+\frac{1}{r}\left\langle y_{x}-z, z-x\right\rangle<0 ;
$$

(B2) $E$ is a bounded set.

Lemma 2.6 (see [39]). Let $E$ be a nonempty closed convex subset of $H$. Let $\phi: E \times E \rightarrow R$ be a bifunction satisfies (A1)-(A5) and let $\varphi: E \rightarrow \mathcal{R} \cup\{+\infty\}$ be a proper lower semicontinuous and convex function. Assume that either (B1) or (B2) holds. For $r>0$ and $x \in H$, define a mapping $T_{r}^{(\phi, \varphi)}: H \rightarrow E$ as follows:

$$
T_{r}^{(\phi, \varphi)}(x)=\left\{z \in E: \phi(z, y)+\varphi(y)-\varphi(z)+\frac{1}{r}\langle y-z, z-x\rangle \geq 0, \forall y \in E\right\}
$$

for all $z \in H$. Then, the following holds:

(i) for each $x \in H, T_{r}^{(\phi, \varphi)}(x) \neq \emptyset$;

(ii) $T_{r}^{(\phi, \varphi)}$ is single-valued;

(iii) $T_{r}^{(\phi, \varphi)}$ is firmly nonexpansive, that is, for any $x, y \in H$,

$$
\left\|T_{r}^{(\phi, \varphi)} x-T_{r}^{(\phi, \varphi)} y\right\|^{2} \leq\left\langle T_{r}^{(\phi, \varphi)} x-T_{r}^{(\phi, \varphi)} y, x-y\right\rangle ;
$$

(iv) $F\left(T_{r}^{(\phi, \varphi)}\right)=\operatorname{MEP}(\phi, \varphi)$;

(v) $\operatorname{MEP}(\phi, \varphi)$ is closed and convex.

Remark 2.7. If $\varphi=0$, then $T_{r}^{(\phi, \varphi)}$ is rewritten as $T_{r}^{\phi}$.

Remark 2.8. We remark that Lemma 2.6 is not a consequence of Lemma 3.1 in [5], because the condition of the sequential continuity from the weak topology to the strong topology for the derivative $K^{\prime}$ of the function $K: E \rightarrow \mathcal{R}$ does not cover the case $K(x)=\|x\|^{2} / 2$.

Lemma 2.9 (see [40]). Let $\left\{x_{n}\right\}$ and $\left\{l_{n}\right\}$ be bounded sequences in a Banach space $X$ and let $\left\{\beta_{n}\right\}$ be a sequence in $[0,1]$ with $0<\liminf _{n \rightarrow \infty} \beta_{n} \leq \lim \sup _{n \rightarrow \infty} \beta_{n}<1$. Suppose $x_{n+1}=\left(1-\beta_{n}\right) l_{n}+\beta_{n} x_{n}$ for all integers $n \geq 1$ and $\lim \sup _{n \rightarrow \infty}\left(\left\|l_{n+1}-l_{n}\right\|-\left\|x_{n+1}-x_{n}\right\|\right) \leq 0$. Then, $\lim _{n \rightarrow \infty}\left\|l_{n}-x_{n}\right\|=0$. 
Fixed Point Theory and Applications

Lemma 2.10 (see [41]). Assume that $\left\{a_{n}\right\}$ is a sequence of nonnegative real numbers such that

$$
a_{n+1} \leq\left(1-\rho_{n}\right) a_{n}+\sigma_{n}, \quad n \geq 1,
$$

where $\left\{\varrho_{n}\right\}$ is a sequence in $(0,1)$ and $\left\{\sigma_{n}\right\}$ is a sequence in $R$ such that

$$
\begin{aligned}
& \text { (1) } \sum_{n=1}^{\infty} \varrho_{n}=\infty \\
& \text { (2) } \lim \sup _{n \rightarrow \infty}\left(\sigma_{n} / \varphi_{n}\right) \leq 0 \text { or } \sum_{n=1}^{\infty}\left|\sigma_{n}\right|<\infty \text {. }
\end{aligned}
$$

Then $\lim _{n \rightarrow \infty} a_{n}=0$.

Lemma 2.11. Let $H$ be a real Hilbert space. Then for all $x, y \in H$,

$$
\|x+y\|^{2} \leq\|x\|^{2}+2\langle y, x+y\rangle \text {. }
$$

\section{Main Results}

In this section, we will use the new approximation iterative method to prove a strong convergence theorem for finding a common element of the set of fixed points of strict pseudocontractions, the set of common solutions of the system of a mixed equilibrium problem and the set of a common solutions of the variational inequalities with inverse strongly monotone mappings in a real Hilbert space.

Theorem 3.1. Let $E$ be a nonempty closed convex subset of a real Hilbert space $H$. Let $\phi_{1}$ and $\phi_{2}$ be two bifunctions from $E \times E$ to $\mathcal{R}$ satisfying (A1)-(A5) and let $\varphi: E \rightarrow \mathcal{R} \cup\{+\infty\}$ be a proper lower semicontinuous and convex function. Let $C: E \rightarrow H$ be an $\xi$-inverse strongly monotone mapping and $B: E \rightarrow H$ be an $\beta$-inverse strongly monotone mapping. Let $f: E \rightarrow E$ be a contraction mapping with coefficient $\alpha(0<\alpha<1)$ and let $A$ be a strongly positive linear bounded operator on $H$ with coefficient $\bar{\gamma}>0$ and $0<\gamma<\bar{\gamma} / \alpha$. Let $S: E \rightarrow E$ be a $k$-strict pseudo-contraction with a fixed point. Define a mapping $S_{k}: E \rightarrow E$ by $S_{k} x=k x+(1-k) S x$, for all $x \in E$. Assume that

$$
\Theta:=F(S) \cap \operatorname{VI}(E, C) \cap \operatorname{VI}(E, B) \cap \operatorname{MEP}\left(\phi_{1}, \varphi\right) \cap \operatorname{MEP}\left(\phi_{2}, \varphi\right) \neq \emptyset .
$$

Assume that either (B1) or (B2). Let $\left\{x_{n}\right\}$ be a sequence generated by the following iterative algorithm:

$$
\begin{gathered}
x_{1} \in E, \quad u_{n} \in E, \quad v_{n} \in E, \\
u_{n}=T_{r}^{\left(\phi_{1}, \varphi\right)} x_{n}, \\
v_{n}=T_{s}^{\left(\phi_{2}, \varphi\right)} x_{n} \\
z_{n}=P_{E}\left(u_{n}-\mu_{n} C u_{n}\right), \\
y_{n}=P_{E}\left(v_{n}-\lambda_{n} B v_{n}\right), \\
k_{n}=a_{n} S_{k} x_{n}+b_{n} y_{n}+c_{n} z_{n}, \\
x_{n+1}=\epsilon_{n} \gamma f\left(x_{n}\right)+\beta_{n} x_{n}+\left(\left(1-\beta_{n}\right) I-\epsilon_{n} A\right) k_{n}, \quad \forall n \geq 1,
\end{gathered}
$$


where $\left\{\epsilon_{n}\right\},\left\{\beta_{n}\right\},\left\{a_{n}\right\},\left\{b_{n}\right\}$, and $\left\{c_{n}\right\}$ are sequences in $(0,1)$ and $\left\{\lambda_{n}\right\},\left\{\mu_{n}\right\}$ are positive sequences. Assume that the control sequences satisfy the following restrictions:

(C1) $a_{n}+b_{n}+c_{n}=1$,

(C2) $\lim _{n \rightarrow \infty} \epsilon_{n}=0$ and $\sum_{n=1}^{\infty} \epsilon_{n}=\infty$,

(C3) $0<\liminf _{n \rightarrow \infty} \beta_{n} \leq \lim \sup _{n \rightarrow \infty} \beta_{n}<1$,

(C4) $\lim _{n \rightarrow \infty}\left|\lambda_{n+1}-\lambda_{n}\right|=\lim _{n \rightarrow \infty}\left|\mu_{n+1}-\mu_{n}\right|=0$,

(C5) $d \leq \lambda_{n} \leq 2 \beta, e \leq \mu_{n} \leq 2 \xi$, where $d$, e are two positive constants,

(C6) $\lim _{n \rightarrow \infty} a_{n}=a, \lim _{n \rightarrow \infty} b_{n}=b$ and $\lim _{n \rightarrow \infty} c_{n}=c$, for some $a, b, c \in(0,1)$.

Then, $\left\{x_{n}\right\}$ converges strongly to a point $q \in \Theta$ which is the unique solution of the variational inequality

$$
\langle(A-\gamma f) q, x-q\rangle \geq 0, \quad \forall x \in \Theta
$$

or equivalent $q=P_{\Theta}(I-A+\gamma f)(q)$, where $P$ is a metric projection mapping form $H$ onto $\Theta$.

Proof. Since $\epsilon_{n} \rightarrow 0$, as $n \rightarrow \infty$, we may assume, without loss of generality, that $\epsilon_{n} \leq(1-$ $\left.\beta_{n}\right)\|A\|^{-1}$ for all $n \in N$. By Lemma 2.2, we know that if $0 \leq \rho \leq\|A\|^{-1}$, then $\|I-\rho A\| \leq 1-\rho \bar{\gamma}$. We will assume that $\|I-A\| \leq 1-\bar{\gamma}$. Since $A$ is a strongly positive bounded linear operator on $H$, we have

$$
\|A\|=\sup \{|\langle A x, x\rangle|: x \in H,\|x\|=1\} .
$$

Observe that

$$
\begin{aligned}
\left\langle\left(\left(1-\beta_{n}\right) I-\epsilon_{n} A\right) x, x\right\rangle & =1-\beta_{n}-\epsilon_{n}\langle A x, x\rangle \\
& \geq 1-\beta_{n}-\epsilon_{n}\|A\| \\
& \geq 0,
\end{aligned}
$$

so this shows that $\left(1-\beta_{n}\right) I-\epsilon_{n} A$ is positive. It follows that

$$
\begin{aligned}
\left\|\left(1-\beta_{n}\right) I-\epsilon_{n} A\right\| & =\sup \left\{\left|\left\langle\left(\left(1-\beta_{n}\right) I-\epsilon_{n} A\right) x, x\right\rangle\right|: x \in H,\|x\|=1\right\} \\
& =\sup \left\{1-\beta_{n}-\epsilon_{n}\langle A x, x\rangle: x \in H,\|x\|=1\right\} \\
& \leq 1-\beta_{n}-\epsilon_{n} \bar{\gamma} .
\end{aligned}
$$

We divide the proof into seven steps.

Step 1. We claim that the mapping $P_{\Theta}(I-A+\gamma f)$ where $\Theta:=F(S) \cap \operatorname{VI}(E, C) \cap \operatorname{VI}(E, B) \cap$ $\operatorname{MEP}\left(\phi_{1}, \varphi\right) \cap \operatorname{MEP}\left(\phi_{2}, \varphi\right)$ has a unique fixed point. 
Since $f$ be a contraction of $H$ into itself with $\alpha \in(0,1)$. Then, we have

$$
\begin{aligned}
\left\|P_{\Theta}(I-A+\gamma f)(x)-P_{\Theta}(I-A+\gamma f)(y)\right\| & \leq\|(I-A+\gamma f)(x)-(I-A+\gamma f)(y)\| \\
& \leq\|I-A\|\|x-y\|+\gamma\|f(x)-f(y)\| \\
& \leq(1-\bar{\gamma})\|x-y\|+\gamma \alpha\|x-y\| \\
& =(1-(\bar{\gamma}-\gamma \alpha))\|x-y\|, \quad \forall x, y \in H .
\end{aligned}
$$

Since $0<1-(\bar{\gamma}-\gamma \alpha)<1$, it follows that $P_{\Theta}(I-A+\gamma f)$ is a contraction of $H$ into itself. Therefore the Banach Contraction Mapping Principle implies that there exists a unique element $q \in H$ such that $q=P_{\Theta}(I-A+\gamma f)(q)$.

Step 2. We claim that $I-\lambda_{n} B$ is nonexpansive.

Indeed, from the $\beta$-inverse strongly monotone mapping definition on $B$ and condition (C5), we have

$$
\begin{aligned}
\left\|\left(I-\lambda_{n} B\right) x-\left(I-\lambda_{n} B\right) y\right\|^{2} & =\left\|(x-y)-\lambda_{n}(B x-B y)\right\|^{2} \\
& =\|x-y\|^{2}-2 \lambda_{n}\langle x-y, B x-B y\rangle+\lambda_{n}^{2}\|B x-B y\|^{2} \\
& \leq\|x-y\|^{2}-2 \lambda_{n} \beta\|B x-B y\|+\lambda_{n}^{2}\|B x-B y\|^{2} \\
& =\|x-y\|^{2}+\lambda_{n}\left(\lambda_{n}-2 \beta\right)\|B x-B y\|^{2} \\
& \leq\|x-y\|^{2},
\end{aligned}
$$

where $\lambda_{n} \leq 2 \beta$, for all $n \in N$ implies that the mapping $I-\lambda_{n} B$ is nonexpansive and so is, $I-\mu_{n} C$.

Step 3. We claim that $\left\{x_{n}\right\}$ is bounded.

Indeed, let $p \in \Theta$ and Lemma 2.6, we obtain

$$
p=P_{E}\left(p-\lambda_{n} B p\right)=P_{E}\left(p-\mu_{n} C p\right)=T_{r}^{\left(\phi_{1}, \varphi\right)} p=T_{s}^{\left(\phi_{2}, \varphi\right)} p .
$$

Note that $u_{n}=T_{r}^{\left(\phi_{1}, \varphi\right)} x_{n} \in \operatorname{dom} \varphi$ and $v_{n}=T_{s}^{\left(\phi_{2}, \varphi\right)} x_{n} \in \operatorname{dom} \varphi$, we have

$$
\begin{aligned}
& \left\|u_{n}-p\right\|=\left\|T_{r}^{\left(\phi_{1}, \varphi\right)} x_{n}-T_{r}^{\left(\phi_{1}, \varphi\right)} p\right\| \leq\left\|x_{n}-p\right\|, \\
& \left\|v_{n}-p\right\|=\left\|T_{s}^{\left(\phi_{2}, \varphi\right)} x_{n}-T_{s}^{\left(\phi_{2}, \varphi\right)} p\right\| \leq\left\|x_{n}-p\right\| .
\end{aligned}
$$


Since $I-\lambda_{n} B$ and $I-\mu_{n} C$ are nonexpansive and from (2.6), we have

$$
\begin{aligned}
\left\|z_{n}-p\right\| & =\left\|P_{E}\left(u_{n}-\mu_{n} C u_{n}\right)-P_{E}\left(p-\mu_{n} C p\right)\right\| \\
& \leq\left\|\left(u_{n}-\mu_{n} C u_{n}\right)-\left(p-\mu_{n} C p\right)\right\| \\
& =\left\|\left(I-\mu_{n} C\right) u_{n}-\left(I-\mu_{n} C\right) p\right\| \\
& \leq\left\|u_{n}-p\right\| \leq\left\|x_{n}-p\right\|, \\
\left\|y_{n}-p\right\| & =\left\|P_{E}\left(v_{n}-\lambda_{n} B v_{n}\right)-P_{E}\left(p-\lambda_{n} B p\right)\right\| \leq\left\|v_{n}-p\right\| \leq\left\|x_{n}-p\right\| .
\end{aligned}
$$

From Lemma 2.3, we have that $S_{k}$ is nonexpansive with $F\left(S_{k}\right)=F(S)$. It follows that

$$
\begin{aligned}
\left\|k_{n}-p\right\| & =\left\|a_{n} S_{k} x_{n}+b_{n} y_{n}+c_{n} z_{n}-p\right\| \\
& \leq a_{n}\left\|S_{k} x_{n}-p\right\|+b_{n}\left\|y_{n}-p\right\|+c_{n}\left\|z_{n}-p\right\| \\
& \leq a_{n}\left\|x_{n}-p\right\|+b_{n}\left\|x_{n}-p\right\|+c_{n}\left\|x_{n}-p\right\|=\left\|x_{n}-p\right\| .
\end{aligned}
$$

It follows that

$$
\begin{aligned}
\left\|x_{n+1}-p\right\| & =\left\|\epsilon_{n}\left(\gamma f\left(x_{n}\right)-A p\right)+\beta_{n}\left(x_{n}-p\right)+\left(\left(1-\beta_{n}\right) I-\epsilon_{n} A\right)\left(k_{n}-p\right)\right\| \\
& \leq\left(1-\beta_{n}-\epsilon_{n} \bar{\gamma}\right)\left\|k_{n}-p\right\|+\beta_{n}\left\|x_{n}-p\right\|+\epsilon_{n}\left\|\gamma f\left(x_{n}\right)-A p\right\| \\
& \leq\left(1-\beta_{n}-\epsilon_{n} \bar{\gamma}\right)\left\|x_{n}-p\right\|+\beta_{n}\left\|x_{n}-p\right\|+\epsilon_{n}\left\|\gamma f\left(x_{n}\right)-A p\right\| \\
& \leq\left(1-\epsilon_{n} \bar{\gamma}\right)\left\|x_{n}-p\right\|+\epsilon_{n} \gamma\left\|f\left(x_{n}\right)-f(p)\right\|+\epsilon_{n}\|\gamma f(p)-A p\| \\
& \leq\left(1-\epsilon_{n} \bar{\gamma}\right)\left\|x_{n}-p\right\|+\epsilon_{n} \gamma \alpha\left\|x_{n}-p\right\|+\epsilon_{n}\|\gamma f(p)-A p\| \\
& =\left(1-(\bar{\gamma}-\alpha \gamma) \epsilon_{n}\right)\left\|x_{n}-p\right\|+(\bar{\gamma}-\alpha \gamma) \epsilon_{n} \frac{\|\gamma f(p)-A p\|}{\bar{\gamma}-\alpha \gamma} \\
& \leq \max \left\{\left\|x_{n}-p\right\|, \frac{\|\gamma f(p)-A p\|}{\bar{\gamma}-\alpha \gamma}\right\} .
\end{aligned}
$$

By simple induction, we have

$$
\left\|x_{n}-p\right\| \leq \max \left\{\left\|x_{1}-p\right\|, \frac{\|\gamma f(p)-A p\|}{\bar{\gamma}-\alpha \gamma}\right\}, \quad \forall n \in N .
$$

Hence, $\left\{x_{n}\right\}$ is bounded, so are $\left\{u_{n}\right\},\left\{v_{n}\right\},\left\{z_{n}\right\},\left\{y_{n}\right\},\left\{k_{n}\right\},\left\{f\left(x_{n}\right)\right\},\left\{C u_{n}\right\}$, and $\left\{B v_{n}\right\}$. 
Step 4. We claim that $\lim _{n \rightarrow \infty}\left\|x_{n+1}-x_{n}\right\|=0$.

Observing that $u_{n}=T_{r}^{\left(\phi_{1}, \varphi\right)} x_{n} \in \operatorname{dom} \varphi$ and $u_{n+1}=T_{r}^{\left(\phi_{1}, \varphi\right)} x_{n+1} \in \operatorname{dom} \varphi$, by the nonexpansiveness of $T_{r}^{\left(\phi_{1}, \varphi\right)}$, we get

$$
\left\|u_{n+1}-u_{n}\right\|=\left\|T_{r}^{\left(\phi_{1}, \varphi\right)} x_{n+1}-T_{r}^{\left(\phi_{1}, \varphi\right)} x_{n}\right\| \leq\left\|x_{n+1}-x_{n}\right\| .
$$

Similarly, let $v_{n}=T_{s}^{\left(\phi_{2}, \varphi\right)} x_{n} \in \operatorname{dom} \varphi$ and $v_{n+1}=T_{s}^{\left(\phi_{2}, \varphi\right)} x_{n+1} \in \operatorname{dom} \varphi$, we have

$$
\left\|v_{n+1}-v_{n}\right\|=\left\|T_{s}^{\left(\phi_{2}, \varphi\right)} x_{n+1}-T_{s}^{\left(\phi_{2}, \varphi\right)} x_{n}\right\| \leq\left\|x_{n+1}-x_{n}\right\| .
$$

From $z_{n}=P_{E}\left(u_{n}-\mu_{n} C u_{n}\right)$ and $y_{n}=P_{E}\left(v_{n}-\lambda_{n} B v_{n}\right)$, we compute

$$
\begin{aligned}
\left\|z_{n+1}-z_{n}\right\| & =\left\|P_{E}\left(u_{n+1}-\mu_{n+1} C u_{n+1}\right)-P_{E}\left(u_{n}-\mu_{n} C u_{n}\right)\right\| \\
& \leq\left\|\left(u_{n+1}-\mu_{n+1} C u_{n+1}\right)-\left(u_{n}-\mu_{n} C u_{n}\right)\right\| \\
& =\left\|\left(u_{n+1}-\mu_{n+1} C u_{n+1}\right)-\left(u_{n}-\mu_{n+1} C u_{n}\right)+\left(\mu_{n}-\mu_{n+1}\right) C u_{n}\right\| \\
& \leq\left\|\left(u_{n+1}-\mu_{n+1} C u_{n+1}\right)-\left(u_{n}-\mu_{n+1} C u_{n}\right)\right\|+\left|\mu_{n}-\mu_{n+1}\right|\left\|C u_{n}\right\| \\
& =\left\|\left(I-\mu_{n+1} C\right) u_{n+1}-\left(I-\mu_{n+1} C\right) u_{n}\right\|+\left|\mu_{n}-\mu_{n+1}\right|\left\|C u_{n}\right\| \\
& \leq\left\|u_{n+1}-u_{n}\right\|+\left|\mu_{n}-\mu_{n+1}\right|\left\|C u_{n}\right\| \\
& \leq\left\|x_{n+1}-x_{n}\right\|+\left|\mu_{n}-\mu_{n+1}\right|\left\|C u_{n}\right\| .
\end{aligned}
$$

Similarly, we have

$$
\begin{aligned}
\left\|y_{n+1}-y_{n}\right\| & =\left\|P_{E}\left(v_{n+1}-\lambda_{n+1} B v_{n+1}\right)-P_{E}\left(v_{n}-\lambda_{n} B v_{n}\right)\right\| \\
& \leq\left\|v_{n+1}-v_{n}\right\|+\left|\lambda_{n}-\lambda_{n+1}\right|\left\|B v_{n}\right\| \\
& \leq\left\|x_{n+1}-x_{n}\right\|+\left|\lambda_{n}-\lambda_{n+1}\right|\left\|B v_{n}\right\| .
\end{aligned}
$$

Observing that

$$
\begin{gathered}
k_{n}=a_{n} S_{k} x_{n}+b_{n} y_{n}+c_{n} z_{n}, \\
k_{n+1}=a_{n+1} S_{k} x_{n+1}+b_{n+1} y_{n+1}+c_{n+1} z_{n+1},
\end{gathered}
$$

we obtain

$$
\begin{aligned}
\left\|k_{n+1}-k_{n}\right\| \leq & a_{n+1}\left\|S_{k} x_{n+1}-S_{k} x_{n}\right\|+\left|a_{n+1}-a_{n}\right|\left\|S_{k} x_{n}\right\|+b_{n+1}\left\|y_{n+1}-y_{n}\right\| \\
& +\left|b_{n+1}-b_{n}\right|\left\|y_{n}\right\|+c_{n+1}\left\|z_{n+1}-z_{n}\right\|+\left|c_{n+1}-c_{n}\right|\left\|z_{n}\right\| \\
\leq & a_{n+1}\left\|x_{n+1}-x_{n}\right\|+\left|a_{n+1}-a_{n}\right|\left\|S_{k} x_{n}\right\|+b_{n+1}\left\|y_{n+1}-y_{n}\right\| \\
& +\left|b_{n+1}-b_{n}\right|\left\|y_{n}\right\|+c_{n+1}\left\|z_{n+1}-z_{n}\right\|+\left|c_{n+1}-c_{n}\right|\left\|z_{n}\right\| .
\end{aligned}
$$


Substituting (3.17) and (3.18) into (3.20), we have

$$
\begin{aligned}
\left\|k_{n+1}-k_{n}\right\| \leq & a_{n+1}\left\|x_{n+1}-x_{n}\right\|+\left|a_{n+1}-a_{n}\right| \mid S_{k} x_{n} \|+b_{n+1}\left\{\left\|x_{n+1}-x_{n}\right\|+\left|\lambda_{n}-\lambda_{n+1}\right|\left\|B v_{n}\right\|\right\} \\
& +c_{n+1}\left\{\left\|x_{n+1}-x_{n}\right\|+\left|\mu_{n}-\mu_{n+1}\right|\left\|C u_{n}\right\|\right\}+\left|b_{n+1}-b_{n}\right|\left\|y_{n}\right\|+\left|c_{n+1}-c_{n}\right|\left\|z_{n}\right\| \\
\leq & \left\|x_{n+1}-x_{n}\right\|+M_{1}\left(\left|a_{n+1}-a_{n}\right|+\left|b_{n+1}-b_{n}\right|+\left|c_{n+1}-c_{n}\right|+\left|\lambda_{n}-\lambda_{n+1}\right|+\left|\mu_{n}-\mu_{n+1}\right|\right)
\end{aligned}
$$

where $M_{1}$ is an appropriate constant such that $M_{1}=\max \left\{\sup _{n \geq 1}\left\|S_{k} x_{n}\right\|,\left\|y_{n}\right\|,\left\|z_{n}\right\|,\left\|B v_{n}\right\|\right.$, $\left.\left\|C u_{n}\right\|\right\}$.

Putting $x_{n+1}=\left(1-\beta_{n}\right) l_{n}+\beta_{n} x_{n}$, for all $n \geq 1$, we have

$$
l_{n}=\frac{x_{n+1}-\beta_{n} x_{n}}{1-\beta_{n}}=\frac{\epsilon_{n} \gamma f\left(x_{n}\right)+\left(\left(1-\beta_{n}\right) I-\epsilon_{n} A\right) k_{n}}{1-\beta_{n}} .
$$

Then, we compute

$$
\begin{aligned}
l_{n+1}-l_{n}= & \frac{\epsilon_{n+1} \gamma f\left(x_{n+1}\right)+\left(\left(1-\beta_{n+1}\right) I-\epsilon_{n+1} A\right) k_{n+1}}{1-\beta_{n+1}} \\
& -\frac{\epsilon_{n} \gamma f\left(x_{n}\right)+\left(\left(1-\beta_{n}\right) I-\epsilon_{n} A\right) k_{n}}{1-\beta_{n}} \\
= & \frac{\epsilon_{n+1}}{1-\beta_{n+1}} \gamma f\left(x_{n+1}\right)-\frac{\epsilon_{n}}{1-\beta_{n}} \gamma f\left(x_{n}\right)+k_{n+1}-k_{n} \\
& +\frac{\epsilon_{n}}{1-\beta_{n}} A k_{n}-\frac{\epsilon_{n+1}}{1-\beta_{n+1}} A k_{n+1} \\
= & \frac{\epsilon_{n+1}}{1-\beta_{n+1}}\left(\gamma f\left(x_{n+1}\right)-A k_{n+1}\right)+\frac{\epsilon_{n}}{1-\beta_{n}}\left(A k_{n}-\gamma f\left(x_{n}\right)\right) \\
& +k_{n+1}-k_{n} .
\end{aligned}
$$

It follows from (3.21) and (3.23), that

$$
\begin{aligned}
\| l_{n+1}- & l_{n}\|-\| x_{n+1}-x_{n} \| \\
\leq & \frac{\epsilon_{n+1}}{1-\beta_{n+1}}\left(\left\|\gamma f\left(x_{n+1}\right)\right\|+\left\|A k_{n+1}\right\|\right)+\frac{\epsilon_{n}}{1-\beta_{n}}\left(\left\|A k_{n}\right\|+\left\|\gamma f\left(x_{n}\right)\right\|\right) \\
& +\left\|k_{n+1}-k_{n}\right\|-\left\|x_{n+1}-x_{n}\right\| \\
\leq & \frac{\epsilon_{n+1}}{1-\beta_{n+1}}\left(\left\|\gamma f\left(x_{n+1}\right)\right\|+\left\|A k_{n+1}\right\|\right)+\frac{\epsilon_{n}}{1-\beta_{n}}\left(\left\|A k_{n}\right\|+\left\|\gamma f\left(x_{n}\right)\right\|\right) \\
& \quad+\mathrm{M}_{1}\left(\left|a_{n+1}-a_{n}\right|+\left|b_{n+1}-b_{n}\right|+\left|c_{n+1}-c_{n}\right|+\left|\lambda_{n}-\lambda_{n+1}\right|+\left|\mu_{n}-\mu_{n+1}\right|\right) .
\end{aligned}
$$


Fixed Point Theory and Applications

This together with (C2), (C3), (C4), and (C6) imply that

$$
\limsup _{n \rightarrow \infty}\left(\left\|l_{n+1}-l_{n}\right\|-\left\|x_{n+1}-x_{n}\right\|\right) \leq 0
$$

Hence, by Lemma 2.9, we obtain $\left\|l_{n}-x_{n}\right\| \rightarrow 0$ as $n \rightarrow \infty$. It follows that

$$
\lim _{n \rightarrow \infty}\left\|x_{n+1}-x_{n}\right\|=\lim _{n \rightarrow \infty}\left(1-\beta_{n}\right)\left\|l_{n}-x_{n}\right\|=0 .
$$

So, we also get

$$
\begin{aligned}
\lim _{n \rightarrow \infty}\left\|u_{n+1}-u_{n}\right\| & =\lim _{n \rightarrow \infty}\left\|v_{n+1}-v_{n}\right\|=\lim _{n \rightarrow \infty}\left\|z_{n+1}-z_{n}\right\| \\
& =\lim _{n \rightarrow \infty}\left\|y_{n+1}-y_{n}\right\|=\lim _{n \rightarrow \infty}\left\|k_{n+1}-k_{n}\right\|=0 .
\end{aligned}
$$

Observe that

$$
x_{n+1}-x_{n}=\epsilon_{n}\left(\gamma f\left(x_{n}\right)-A x_{n}\right)+\left(1-\beta_{n}-\epsilon_{n} \bar{\gamma}\right)\left(k_{n}-x_{n}\right) .
$$

By condition (C2) and (3.26), we have

$$
\lim _{n \rightarrow \infty}\left\|k_{n}-x_{n}\right\|=0 .
$$

Step 5. We claim that the following statements hold:

(s1) $\lim _{n \rightarrow \infty}\left\|x_{n}-v_{n}\right\|=0$;

(s2) $\lim _{n \rightarrow \infty}\left\|x_{n}-u_{n}\right\|=0$;

(s3) $\lim _{n \rightarrow \infty}\left\|x_{n}-y_{n}\right\|=0$;

(s4) $\lim _{n \rightarrow \infty}\left\|x_{n}-z_{n}\right\|=0$.

Indeed, pick any $p \in \Theta$, to obtain

$$
\begin{aligned}
\left\|u_{n}-p\right\|^{2} & =\left\|T_{r}^{\left(\phi_{1}, \varphi\right)} x_{n}-T_{r}^{\left(\phi_{1}, \varphi\right)} p\right\|^{2} \\
& \leq\left\langle T_{r}^{\left(\phi_{1}, \varphi\right)} x_{n}-T_{r}^{\left(\phi_{1}, \varphi\right)} p, x_{n}-p\right\rangle \\
& =\left\langle u_{n}-p, x_{n}-p\right\rangle \\
& =\frac{1}{2}\left(\left\|u_{n}-p\right\|^{2}+\left\|x_{n}-p\right\|^{2}-\left\|x_{n}-u_{n}\right\|^{2}\right) .
\end{aligned}
$$

Therefore,

$$
\left\|u_{n}-p\right\|^{2} \leq\left\|x_{n}-p\right\|^{2}-\left\|x_{n}-u_{n}\right\|^{2} .
$$


Similarly, we have

$$
\left\|v_{n}-p\right\|^{2} \leq\left\|x_{n}-p\right\|^{2}-\left\|x_{n}-v_{n}\right\|^{2} .
$$

Note that

$$
\begin{aligned}
\left\|k_{n}-p\right\|^{2} & =\left\|a_{n} S_{k} x_{n}+b_{n} y_{n}+c_{n} z_{n}-p\right\|^{2} \\
& \leq a_{n}\left\|S_{k} x_{n}-p\right\|^{2}+b_{n}\left\|y_{n}-p\right\|^{2}+c_{n}\left\|z_{n}-p\right\|^{2} \\
& \leq a_{n}\left\|x_{n}-p\right\|^{2}+b_{n}\left\|v_{n}-p\right\|+c_{n}\left\|u_{n}-p\right\| .
\end{aligned}
$$

Substituting (3.31) and (3.32) into (3.33), we obtain

$$
\begin{aligned}
\left\|k_{n}-p\right\|^{2} & \leq a_{n}\left\|x_{n}-p\right\|^{2}+b_{n}\left\|v_{n}-p\right\|+c_{n}\left\|u_{n}-p\right\| \\
& \leq a_{n}\left\|x_{n}-p\right\|^{2}+b_{n}\left\{\left\|x_{n}-p\right\|^{2}-\left\|x_{n}-v_{n}\right\|^{2}\right\}+c_{n}\left\{\left\|x_{n}-p\right\|^{2}-\left\|x_{n}-u_{n}\right\|^{2}\right\} \\
& =\left\|x_{n}-p\right\|^{2}-b_{n}\left\|x_{n}-v_{n}\right\|^{2}-c_{n}\left\|x_{n}-u_{n}\right\|^{2} .
\end{aligned}
$$

From Lemma 2.1, (3.2) and (3.34), we obtain

$$
\begin{aligned}
\left\|x_{n+1}-p\right\|^{2}= & \left\|\epsilon_{n}\left(\gamma f\left(x_{n}\right)-A p\right)+\beta_{n}\left(x_{n}-p\right)+\left(\left(1-\beta_{n}\right) I-\epsilon_{n} A\right)\left(k_{n}-p\right)\right\|^{2} \\
\leq & \epsilon_{n}\left\|\gamma f\left(x_{n}\right)-A p\right\|^{2}+\beta_{n}\left\|x_{n}-p\right\|^{2}+\left(1-\beta_{n}-\epsilon_{n} \bar{\gamma}\right)\left\|k_{n}-p\right\|^{2} \\
\leq & \epsilon_{n}\left\|\gamma f\left(x_{n}\right)-A p\right\|^{2}+\beta_{n}\left\|x_{n}-p\right\|^{2} \\
& +\left(1-\beta_{n}-\epsilon_{n} \bar{\gamma}\right)\left\{\left\|x_{n}-p\right\|^{2}-b_{n}\left\|x_{n}-v_{n}\right\|^{2}-c_{n}\left\|x_{n}-u_{n}\right\|^{2}\right\} \\
= & \epsilon_{n}\left\|\gamma f\left(x_{n}\right)-A p\right\|^{2}+\left(1-\epsilon_{n} \bar{\gamma}\right)\left\|x_{n}-p\right\|^{2}-\left(1-\beta_{n}-\epsilon_{n} \bar{\gamma}\right) b_{n}\left\|x_{n}-v_{n}\right\|^{2} \\
& -\left(1-\beta_{n}-\epsilon_{n} \bar{\gamma}\right) c_{n}\left\|x_{n}-u_{n}\right\|^{2} \\
\leq & \epsilon_{n}\left\|\gamma f\left(x_{n}\right)-A p\right\|^{2}+\left\|x_{n}-p\right\|^{2}-\left(1-\beta_{n}-\epsilon_{n} \bar{\gamma}\right) b_{n}\left\|x_{n}-v_{n}\right\|^{2} \\
& -\left(1-\beta_{n}-\epsilon_{n} \bar{\gamma}\right) c_{n}\left\|x_{n}-u_{n}\right\|^{2} .
\end{aligned}
$$

It follows that

$$
\begin{aligned}
\left(1-\beta_{n}-\epsilon_{n} \bar{\gamma}\right) c_{n}\left\|x_{n}-u_{n}\right\|^{2} & \leq \epsilon_{n}\left\|\gamma f\left(x_{n}\right)-A p\right\|^{2}+\left\|x_{n}-p\right\|^{2}-\left\|x_{n+1}-p\right\|^{2} \\
& \leq \epsilon_{n}\left\|\gamma f\left(x_{n}\right)-A p\right\|^{2}+\left\|x_{n+1}-x_{n}\right\|\left(\left\|x_{n}-p\right\|+\left\|x_{n+1}-p\right\|\right)
\end{aligned}
$$


Fixed Point Theory and Applications

From (C2), (C6), and (3.26), we also have

$$
\lim _{n \rightarrow \infty}\left\|x_{n}-u_{n}\right\|=0
$$

Similarly, using (3.35) again, we have

$$
\begin{aligned}
\left(1-\beta_{n}-\epsilon_{n} \bar{\gamma}\right) b_{n}\left\|x_{n}-v_{n}\right\|^{2} & \leq \epsilon_{n}\left\|\gamma f\left(x_{n}\right)-A p\right\|^{2}+\left\|x_{n}-p\right\|^{2}-\left\|x_{n+1}-p\right\|^{2} \\
& \leq \epsilon_{n}\left\|\gamma f\left(x_{n}\right)-A p\right\|^{2}+\left\|x_{n+1}-x_{n}\right\|\left(\left\|x_{n}-p\right\|+\left\|x_{n+1}-p\right\|\right)
\end{aligned}
$$

From (C2), (C6), and (3.26), we also have

$$
\lim _{n \rightarrow \infty}\left\|x_{n}-v_{n}\right\|=0
$$

From (3.37) and (3.39), we have

$$
\lim _{n \rightarrow \infty}\left\|u_{n}-v_{n}\right\|=0
$$

For $p \in \Theta$, we compute

$$
\begin{aligned}
\left\|z_{n}-p\right\|^{2} & =\left\|P_{E}\left(u_{n}-\mu_{n} C u_{n}\right)-P_{E}\left(p-\mu_{n} C p\right)\right\|^{2} \\
& \leq\left\|\left(u_{n}-\mu_{n} C u_{n}\right)-\left(p-\mu_{n} C p\right)\right\|^{2} \\
& =\left\|\left(u_{n}-p\right)-\mu_{n}\left(C u_{n}-C p\right)\right\|^{2} \\
& \leq\left\|u_{n}-p\right\|^{2}-2 \mu_{n}\left\langle u_{n}-p, C u_{n}-C p\right\rangle+\mu_{n}^{2}\left\|C u_{n}-C p\right\|^{2} \\
& \leq\left\|x_{n}-p\right\|^{2}+\mu_{n}\left(\mu_{n}-2 \xi\right)\left\|C u_{n}-C p\right\|^{2} \\
& \leq\left\|x_{n}-p\right\|^{2}-\mu_{n}\left(2 \xi-\mu_{n}\right)\left\|C u_{n}-C p\right\|^{2} .
\end{aligned}
$$

Similarly, we have

$$
\left\|y_{n}-p\right\|^{2} \leq\left\|x_{n}-p\right\|^{2}-\lambda_{n}\left(2 \beta-\lambda_{n}\right)\left\|B v_{n}-B p\right\|^{2} .
$$


Substituting (3.41) and (3.42) into (3.33), we also have

$$
\begin{aligned}
\left\|k_{n}-p\right\|^{2} \leq & a_{n}\left\|S_{k} x_{n}-p\right\|^{2}+b_{n}\left\|y_{n}-p\right\|^{2}+c_{n}\left\|z_{n}-p\right\|^{2} \\
\leq & a_{n}\left\|x_{n}-p\right\|^{2}+b_{n}\left\{\left\|x_{n}-p\right\|^{2}-\lambda_{n}\left(2 \beta-\lambda_{n}\right)\left\|B v_{n}-B p\right\|^{2}\right\} \\
& +c_{n}\left\{\left\|x_{n}-p\right\|^{2}-\mu_{n}\left(2 \xi-\mu_{n}\right)\left\|C u_{n}-C p\right\|^{2}\right\} \\
= & \left\|x_{n}-p\right\|^{2}-b_{n} \lambda_{n}\left(2 \beta-\lambda_{n}\right)\left\|B v_{n}-B p\right\|^{2}-c_{n} \mu_{n}\left(2 \xi-\mu_{n}\right)\left\|C u_{n}-C p\right\|^{2} .
\end{aligned}
$$

On the other hand, we note that

$$
\begin{aligned}
&\left\|x_{n+1}-p\right\|^{2} \\
& \leq \epsilon_{n}\left\|\gamma f\left(x_{n}\right)-A p\right\|^{2}+\beta_{n}\left\|x_{n}-p\right\|^{2}+\left(1-\beta_{n}-\epsilon_{n} \bar{\gamma}\right)\left\|k_{n}-p\right\|^{2} \\
& \leq \epsilon_{n}\left\|\gamma f\left(x_{n}\right)-A p\right\|^{2}+\beta_{n}\left\|x_{n}-p\right\|^{2} \\
&+\left(1-\beta_{n}-\epsilon_{n} \bar{\gamma}\right)\left\{\left\|x_{n}-p\right\|^{2}-b_{n} \lambda_{n}\left(2 \beta-\lambda_{n}\right)\left\|B v_{n}-B p\right\|^{2}-c_{n} \mu_{n}\left(2 \xi-\mu_{n}\right)\left\|C u_{n}-C p\right\|^{2}\right\} \\
&= \epsilon_{n}\left\|\gamma f\left(x_{n}\right)-A p\right\|^{2}+\left(1-\epsilon_{n} \bar{\gamma}\right)\left\|x_{n}-p\right\|^{2}-\left(1-\beta_{n}-\epsilon_{n} \bar{\gamma}\right) b_{n} \lambda_{n}\left(2 \beta-\lambda_{n}\right)\left\|B v_{n}-B p\right\|^{2} \\
&-\left(1-\beta_{n}-\epsilon_{n} \bar{\gamma}\right) c_{n} \mu_{n}\left(2 \xi-\mu_{n}\right)\left\|C u_{n}-C p\right\|^{2} \\
& \leq \epsilon_{n}\left\|\gamma f\left(x_{n}\right)-A p\right\|^{2}+\left\|x_{n}-p\right\|^{2}-\left(1-\beta_{n}-\epsilon_{n} \bar{\gamma}\right) b_{n} \lambda_{n}\left(2 \beta-\lambda_{n}\right)\left\|B v_{n}-B p\right\|^{2} \\
&-\left(1-\beta_{n}-\epsilon_{n} \bar{\gamma}\right) c_{n} \mu_{n}\left(2 \xi-\mu_{n}\right)\left\|C u_{n}-C p\right\|^{2} .
\end{aligned}
$$

It follows that

$$
\begin{aligned}
\left(1-\beta_{n}-\epsilon_{n} \bar{\gamma}\right) c_{n} \mu_{n}\left(2 \xi-\mu_{n}\right)\left\|C u_{n}-C p\right\|^{2} \\
\quad \leq \epsilon_{n}\left\|\gamma f\left(x_{n}\right)-A p\right\|^{2}+\left\|x_{n}-p\right\|^{2}-\left\|x_{n+1}-p\right\|^{2} \\
\quad \leq \epsilon_{n}\left\|\gamma f\left(x_{n}\right)-A p\right\|^{2}+\left\|x_{n+1}-x_{n}\right\|\left(\left\|x_{n}-p\right\|+\left\|x_{n+1}-p\right\|\right)
\end{aligned}
$$

From (C2), (C5), (C6), and (3.26), we have

$$
\lim _{n \rightarrow \infty}\left\|C u_{n}-C p\right\|=0
$$

Thanks to (3.44), we also have

$$
\begin{aligned}
& \left(1-\beta_{n}-\epsilon_{n} \bar{\gamma}\right) b_{n} \lambda_{n}\left(2 \beta-\lambda_{n}\right)\left\|B v_{n}-B p\right\|^{2} \\
& \quad \leq \epsilon_{n}\left\|\gamma f\left(x_{n}\right)-A p\right\|^{2}+\left\|x_{n+1}-x_{n}\right\|\left(\left\|x_{n}-p\right\|+\left\|x_{n+1}-p\right\|\right) .
\end{aligned}
$$


Fixed Point Theory and Applications

From (C2), (C5), (C6), and (3.26), we obtain

$$
\lim _{n \rightarrow \infty}\left\|B v_{n}-B p\right\|=0
$$

Observe that

$$
\begin{aligned}
\| y_{n}- & p \|^{2} \\
& =\left\|P_{E}\left(v_{n}-\lambda_{n} B v_{n}\right)-P_{E}\left(p-\lambda_{n} B p\right)\right\|^{2} \\
& \leq\left\langle\left(I-\lambda_{n} B\right) v_{n}-\left(I-\lambda_{n} B\right) p, y_{n}-p\right\rangle \\
& =\frac{1}{2}\left\{\left\|\left(I-\lambda_{n} B\right) v_{n}-\left(I-\lambda_{n} B\right) p\right\|^{2}+\left\|y_{n}-p\right\|^{2}-\left\|\left(I-\lambda_{n} B\right) v_{n}-\left(I-\lambda_{n} B\right) p-\left(y_{n}-p\right)\right\|^{2}\right\} \\
& \leq \frac{1}{2}\left\{\left\|v_{n}-p\right\|^{2}+\left\|y_{n}-p\right\|^{2}-\left\|\left(v_{n}-y_{n}\right)-\lambda_{n}\left(B v_{n}-B p\right)\right\|^{2}\right\} \\
& \leq \frac{1}{2}\left\{\left\|x_{n}-p\right\|^{2}+\left\|y_{n}-p\right\|^{2}-\left\|v_{n}-y_{n}\right\|^{2}-\lambda_{n}^{2}\left\|B v_{n}-B p\right\|^{2}+2 \lambda_{n}\left\langle v_{n}-y_{n}, B v_{n}-B p\right\rangle\right\},
\end{aligned}
$$

and hence

$$
\left\|y_{n}-p\right\|^{2} \leq\left\|x_{n}-p\right\|^{2}-\left\|v_{n}-y_{n}\right\|^{2}+2 \lambda_{n}\left\|v_{n}-y_{n}\right\|\left\|B v_{n}-B p\right\| .
$$

Similarly, we can obtain that

$$
\left\|z_{n}-p\right\|^{2} \leq\left\|x_{n}-p\right\|^{2}-\left\|u_{n}-z_{n}\right\|^{2}+2 \mu_{n}\left\|u_{n}-z_{n}\right\|\left\|C u_{n}-C p\right\| .
$$

Substituting (3.50) and (3.51) into (3.33), we also have

$$
\begin{aligned}
\left\|k_{n}-p\right\|^{2} \leq & a_{n}\left\|S_{k} x_{n}-p\right\|^{2}+b_{n}\left\|y_{n}-p\right\|^{2}+c_{n}\left\|z_{n}-p\right\|^{2} \\
\leq & a_{n}\left\|x_{n}-p\right\|^{2}+b_{n}\left\{\left\|x_{n}-p\right\|^{2}-\left\|v_{n}-y_{n}\right\|^{2}+2 \lambda_{n}\left\|v_{n}-y_{n}\right\|\left\|B v_{n}-B p\right\|\right\} \\
& +c_{n}\left\{\left\|x_{n}-p\right\|^{2}-\left\|u_{n}-z_{n}\right\|^{2}+2 \mu_{n}\left\|u_{n}-z_{n}\right\|\left\|C u_{n}-C p\right\|\right\} \\
= & \left\|x_{n}-p\right\|^{2}-b_{n}\left\|v_{n}-y_{n}\right\|^{2}+2 b_{n} \lambda_{n}\left\|v_{n}-y_{n}\right\|\left\|B v_{n}-B p\right\| \\
& -c_{n}\left\|u_{n}-z_{n}\right\|^{2}+2 \mu_{n}\left\|u_{n}-z_{n}\right\|\left\|C u_{n}-C p\right\| .
\end{aligned}
$$


On the other hand, we have

$$
\begin{aligned}
\left\|x_{n+1}-p\right\|^{2} \leq & \epsilon_{n}\left\|\gamma f\left(x_{n}\right)-A p\right\|^{2}+\beta_{n}\left\|x_{n}-p\right\|^{2}+\left(1-\beta_{n}-\epsilon_{n} \bar{\gamma}\right)\left\|k_{n}-p\right\|^{2} \\
\leq & \epsilon_{n}\left\|\gamma f\left(x_{n}\right)-A p\right\|^{2}+\beta_{n}\left\|x_{n}-p\right\|^{2} \\
& +\left(1-\beta_{n}-\epsilon_{n} \bar{\gamma}\right)\left\{\left\|x_{n}-p\right\|^{2}-b_{n}\left\|v_{n}-y_{n}\right\|^{2}+2 b_{n} \lambda_{n}\left\|v_{n}-y_{n}\right\|\left\|B v_{n}-B p\right\|\right. \\
\left.\quad-c_{n}\left\|u_{n}-z_{n}\right\|^{2}+2 \mu_{n}\left\|u_{n}-z_{n}\right\|\left\|C u_{n}-C p\right\|\right\} & \\
= & \epsilon_{n}\left\|\gamma f\left(x_{n}\right)-A p\right\|^{2}+\left(1-\epsilon_{n} \bar{\gamma}\right)\left\|x_{n}-p\right\|^{2}-\left(1-\beta_{n}-\epsilon_{n} \bar{\gamma}\right) b_{n}\left\|v_{n}-y_{n}\right\|^{2} \\
& +2 b_{n}\left(1-\beta_{n}-\epsilon_{n} \bar{\gamma}\right) \lambda_{n}\left\|v_{n}-y_{n}\right\|\left\|B v_{n}-B p\right\|-\left(1-\beta_{n}-\epsilon_{n} \bar{\gamma}\right) c_{n}\left\|u_{n}-z_{n}\right\|^{2} \\
& +2 c_{n}\left(1-\beta_{n}-\epsilon_{n} \bar{\gamma}\right) \mu_{n}\left\|u_{n}-z_{n}\right\|\left\|C u_{n}-C p\right\| \\
\leq & \epsilon_{n}\left\|\gamma f\left(x_{n}\right)-A p\right\|^{2}+\left\|x_{n}-p\right\|^{2}-\left(1-\beta_{n}-\epsilon_{n} \bar{\gamma}\right) b_{n}\left\|v_{n}-y_{n}\right\|^{2} \\
& +2 b_{n}\left(1-\beta_{n}-\epsilon_{n} \bar{\gamma}\right) \lambda_{n}\left\|v_{n}-y_{n}\right\|\left\|B v_{n}-B p\right\|-\left(1-\beta_{n}-\epsilon_{n} \bar{\gamma}\right) c_{n}\left\|u_{n}-z_{n}\right\|^{2} \\
& +2 c_{n}\left(1-\beta_{n}-\epsilon_{n} \bar{\gamma}\right) \mu_{n}\left\|u_{n}-z_{n}\right\|\left\|C u_{n}-C p\right\|
\end{aligned}
$$

and hence

$$
\begin{aligned}
\left(1-\beta_{n}-\epsilon_{n} \bar{\gamma}\right) b_{n}\left\|v_{n}-y_{n}\right\|^{2} \leq & \epsilon_{n}\left\|\gamma f\left(x_{n}\right)-A p\right\|^{2}+\left\|x_{n}-p\right\|^{2}-\left\|x_{n+1}-p\right\|^{2} \\
& +2 b_{n}\left(1-\beta_{n}-\epsilon_{n} \bar{\gamma}\right) \lambda_{n}\left\|v_{n}-y_{n}\right\|\left\|B v_{n}-B p\right\| \\
& +2 c_{n}\left(1-\beta_{n}-\epsilon_{n} \bar{\gamma}\right) \mu_{n}\left\|u_{n}-z_{n}\right\|\left\|C u_{n}-C p\right\| \\
\leq & \epsilon_{n}\left\|\gamma f\left(x_{n}\right)-A p\right\|^{2}+\left\|x_{n+1}-x_{n}\right\|\left(\left\|x_{n}-p\right\|+\left\|x_{n+1}-p\right\|\right) \\
& +2 b_{n}\left(1-\beta_{n}-\epsilon_{n} \bar{\gamma}\right) \lambda_{n}\left\|v_{n}-y_{n}\right\|\left\|B v_{n}-B p\right\| \\
& +2 c_{n}\left(1-\beta_{n}-\epsilon_{n} \bar{\gamma}\right) \mu_{n}\left\|u_{n}-z_{n}\right\|\left\|C u_{n}-C p\right\| .
\end{aligned}
$$

From (C2), (C6), (3.26), (3.46), and (3.48), we also have

$$
\lim _{n \rightarrow \infty}\left\|v_{n}-y_{n}\right\|=0
$$


Fixed Point Theory and Applications

Similarly, using (3.53) again, we can prove

$$
\lim _{n \rightarrow \infty}\left\|u_{n}-z_{n}\right\|=0
$$

From (3.39) and (3.55), we also have

$$
\lim _{n \rightarrow \infty}\left\|x_{n}-y_{n}\right\|=0
$$

From (3.37) and (3.56), we have

$$
\lim _{n \rightarrow \infty}\left\|x_{n}-z_{n}\right\|=0
$$

Step 6. We claim that limsup $\sup _{n \rightarrow \infty}\left\langle(A-\gamma f) q, q-x_{n}\right\rangle \leq 0$, where $q=P_{\Theta}(I-A+\gamma f)(q)$ is the unique solution of the variational inequality $\langle(A-\gamma f) q, x-q\rangle \geq 0$, for all $x \in \Theta$.

To show this inequality, we choose a subsequence $\left\{x_{n_{i}}\right\}$ of $\left\{x_{n}\right\}$ such that

$$
\limsup _{n \rightarrow \infty}\left\langle(A-\gamma f) q, q-x_{n}\right\rangle=\lim _{i \rightarrow \infty}\left\langle(A-\gamma f) q, q-x_{n_{i}}\right\rangle
$$

Since $\left\{x_{n_{i}}\right\}$ is bounded, there exists a subsequence $\left\{x_{n_{i_{j}}}\right\}$ of $\left\{x_{n_{i}}\right\}$ which converges weakly to $z \in E$. Without loss of generality, we can assume that $x_{n_{i}} \rightarrow z$. We claim that $z \in \Theta$.

(a1) First, we prove that $z \in F(S) \cap \operatorname{VI}(E, C) \cap \operatorname{VI}(E, B)$.

Assume also that $\lambda_{n} \rightarrow \lambda \in[d, 2 \beta]$ and $\mu_{n} \rightarrow \mu \in[e, 2 \xi]$.

Define a mapping $\Omega: E \rightarrow E$ by

$$
\Omega x=a S_{k} x+b P_{E}(1-\mu C) x+c P_{E}(1-\lambda B) x, \quad \forall x \in E,
$$

where $\lim _{n \rightarrow \infty} a_{n}=a, \lim _{n \rightarrow \infty} b_{n}=b$, and $\lim _{n \rightarrow \infty} c_{n}=c$, for some $a, b, c \in(0,1)$. From Lemma 2.5, we have that $\Omega$ is nonexpansive with

$$
F(\Omega)=F\left(S_{k}\right) \cap F\left(P_{E}(1-\mu C)\right) \cap F\left(P_{E}(1-\lambda B)\right)=F(S) \cap \operatorname{VI}(E, C) \cap \operatorname{VI}(E, B) .
$$


Notice that

$$
\begin{aligned}
\| \Omega x_{n}- & x_{n} \| \\
\leq & \left\|\Omega x_{n}-k_{n}\right\|+\left\|k_{n}-x_{n}\right\| \\
\leq & \left\|\left[a S_{k} x_{n}+b P_{E}(1-\lambda B) x_{n}+c P_{E}(1-\mu C) x_{n}\right]-\left[a_{n} S_{k} x_{n}+b_{n} y_{n}+c_{n} z_{n}\right]\right\|+\left\|k_{n}-x_{n}\right\| \\
\leq & \left|a-a_{n}\right|\left\|S_{k} x_{n}\right\|+\left\|b P_{E}(I-\lambda B) x_{n}-b_{n} P_{E}\left(I-\lambda_{n} B\right) x_{n}\right\| \\
& +\left\|b_{n} P_{E}\left(I-\lambda_{n} B\right) x_{n}-b_{n} P_{E}\left(I-\lambda_{n} B\right) v_{n}\right\|+\left\|c P_{E}(I-\mu C) x_{n}-c_{n} P_{E}\left(1-\mu_{n} C\right) x_{n}\right\| \\
& +\left\|c_{n} P_{E}\left(I-\mu_{n} B\right) x_{n}-c_{n} P_{E}\left(I-\mu_{n} C\right) u_{n}\right\|+\left\|k_{n}-x_{n}\right\| \\
\leq & \left|a-a_{n}\right|\left\|S_{k} x_{n}\right\|+\left|b-b_{n}\right|\left\|x_{n}\right\|+\left|b_{n} \lambda_{n}-b \lambda\right|\left\|B x_{n}\right\|+\left|c-c_{n}\right|\left\|x_{n}\right\|+\left|c_{n} \mu_{n}-c \mu\right|\left\|C x_{n}\right\| \\
& +b_{n}\left\|x_{n}-v_{n}\right\|+c_{n}\left\|x_{n}-u_{n}\right\|+\left\|k_{n}-x_{n}\right\| \\
\leq & K_{1}\left(\left|a-a_{n}\right|+\left|b-b_{n}\right|+\left|c-c_{n}\right|+\left|b \lambda-b_{n} \lambda_{n}\right|+\left|c \mu-c_{n} \mu_{n}\right|\right) \\
& +b_{n}\left\|x_{n}-v_{n}\right\|+c_{n}\left\|x_{n}-u_{n}\right\|+\left\|k_{n}-x_{n}\right\|,
\end{aligned}
$$

where $K_{1}$ is an appropriate constant such that

$$
K_{1}=\max \left\{\sup _{n \geq 1}\left\|x_{n}\right\|, \sup _{n \geq 1}\left\|B x_{n}\right\|, \sup _{n \geq 1}\left\|C x_{n}\right\|, \sup _{n \geq 1}\left\|S_{k} x_{n}\right\|\right\} .
$$

From (C6), (3.37), (3.39), and (3.29), we obtain

$$
\lim _{n \rightarrow \infty}\left\|x_{n}-\Omega x_{n}\right\|=0
$$

By Lemma 2.4, we have $z \in F(\Omega)$, that is, $z \in F(S) \cap \operatorname{VI}(E, C) \cap \operatorname{VI}(E, B)$.

(a2) Now, we prove that $z \in F(S) \cap \operatorname{MEP}\left(\phi_{1}, \varphi\right) \cap \operatorname{MEP}\left(\phi_{2}, \varphi\right)$.

Define a mapping $Q: E \rightarrow E$ by

$$
Q x=a S_{k} x+b T_{r}^{\left(\phi_{1}, \varphi\right)} x+c T_{S}^{\left(\phi_{2}, \varphi\right)} x, \quad \forall x \in E,
$$

where $\lim _{n \rightarrow \infty} a_{n}=a, \lim _{n \rightarrow \infty} b_{n}=b$, and $\lim _{n \rightarrow \infty} c_{n}=c$, for some $a, b, c \in(0,1)$. From Lemma 2.5, we have that $Q$ is nonexpansive with

$$
F(Q)=F\left(S_{k}\right) \cap F\left(T_{r}^{\left(\phi_{1}, \varphi\right)}\right) \cap F\left(T_{s}^{\left(\phi_{2}, \varphi\right)}\right)=F(S) \cap \operatorname{MEP}\left(\phi_{1}, \varphi\right) \cap\left(\phi_{2}, \varphi\right) .
$$


On the other hand, we have

$$
\begin{aligned}
\left\|Q x_{n}-x_{n}\right\| \leq & \left\|Q x_{n}-k_{n}\right\|+\left\|k_{n}-x_{n}\right\| \\
\leq & \left\|\left[a S_{k} x_{n}+b T_{r}^{\left(\phi_{1}, \varphi\right)} x_{n}+c T_{s}^{\left(\phi_{2}, \varphi\right)} x_{n}\right]-\left[a_{n} S_{k} x_{n}+b_{n} y_{n}+c_{n} z_{n}\right]\right\|+\left\|k_{n}-x_{n}\right\| \\
\leq & \left|a-a_{n}\right|\left\|S_{k} x_{n}\right\|+|b|\left\|T_{r}^{\left(\phi_{1}, \varphi\right)} x_{n}\right\|+|c|\left\|T_{s}^{\left(\phi_{2}, \varphi\right)} x_{n}\right\| \\
& +\left|b_{n}-b\right|\left\|P_{E}\left(I-\lambda_{n} B\right) v_{n}\right\|+\left|c_{n}-c\right|\left\|P_{E}\left(I-\mu_{n} C\right) u_{n}\right\| \\
& +|b|\left\|P_{E}\left(I-\lambda_{n} B\right) v_{n}\right\|+|c|\left\|P_{E}\left(I-\mu_{n} C\right) u_{n}\right\|+\left\|k_{n}-x_{n}\right\| \\
\leq & K_{2}\left(\left|a-a_{n}\right|+\left|b-b_{n}\right|+\left|c-c_{n}\right|+2|b|+2|c|\right)+\left\|k_{n}-x_{n}\right\|,
\end{aligned}
$$

where $K_{2}$ is an appropriate constant such that

$$
\begin{aligned}
& K_{2}=\max \left\{\sup _{n \geq 1}\left\|T_{r}^{\left(\phi_{1}, \varphi\right)} x_{n}\right\|, \sup _{n \geq 1}\left\|T_{s}^{\left(\phi_{2}, \varphi\right)} x_{n}\right\|, \sup _{n \geq 1}\left\|S_{k} x_{n}\right\|,\right. \\
&\left.\sup _{n \geq 1}\left\{\left\|T_{r}^{\left(\phi_{1}, \varphi\right)} x_{n}\right\|+\left\|P_{E}\left(I-\lambda_{n} B\right) v_{n}\right\|\right\}, \sup _{n \geq 1}\left\{\left\|T_{s}^{\left(\phi_{2}, \varphi\right)} x_{n}\right\|+\left\|P_{E}\left(I-\mu_{n} C\right) u_{n}\right\|\right\}\right\} .
\end{aligned}
$$

From (C6) and (3.29), we obtain

$$
\lim _{n \rightarrow \infty}\left\|x_{n}-Q x_{n}\right\|=0
$$

Since $P_{\Theta}(I-A+\gamma f)(q)$ is a contraction with the coefficient $\alpha \in(0,1)$, there exists a unique fixed point. We use $q$ to denote the unique fixed point to the mapping $P_{\Theta}(I-A+\gamma f)(q)$, that is, $q=P_{\Theta}(I-A+\gamma f)(q)$. Since $\left\{x_{n_{i}}\right\}$ is bounded, There exists a subsequence $\left\{x_{n_{i}}\right\}$ of $\left\{x_{n}\right\}$ which converges weakly to $z$. Without loss of generality, we may assume that $\left\{x_{n_{i}}\right\} \rightarrow z$. It follows from (3.69), that

$$
\lim _{n \rightarrow \infty}\left\|x_{n_{i}}-Q x_{n_{i}}\right\|=0
$$

It follows from Lemma 2.4, we obtain that $z \in F(Q)$. Hence $z \in \Theta$, where $\Theta:=F(S) \cap$ $\operatorname{VI}(E, C) \cap \operatorname{VI}(E, B) \cap \operatorname{MEP}\left(\phi_{1}, \varphi\right) \cap \operatorname{MEP}\left(\phi_{2}, \varphi\right)$. From (3.59) and (2.4), we arrive at

$$
\begin{aligned}
\limsup _{n \rightarrow \infty}\left\langle(A-\gamma f) q, q-x_{n}\right\rangle & =\limsup _{n \rightarrow \infty}\left\langle(A-\gamma f) q, q-x_{n_{i}}\right\rangle \\
& =\langle(A-\gamma f) q, q-z\rangle \leq 0 .
\end{aligned}
$$


On the other hand, we have

$$
\begin{aligned}
\left\langle(A-\gamma f) q, q-x_{n+1}\right\rangle & =\left\langle(A-\gamma f) q, x_{n}-x_{n+1}\right\rangle+\left\langle(A-\gamma f) q, q-x_{n}\right\rangle \\
& \leq\|(A-\gamma f) q\|\left\|x_{n}-x_{n+1}\right\|+\left\langle(A-\gamma f) q, q-x_{n}\right\rangle .
\end{aligned}
$$

From (3.26) and (3.71), we obtain that

$$
\limsup _{n \rightarrow \infty}\left\langle(A-\gamma f) q, q-x_{n+1}\right\rangle \leq 0 .
$$

Step 7. We claim that $\lim _{n \rightarrow \infty}\left\|x_{n}-q\right\|=0$.

Indeed, by (3.2) and using Lemmas 2.2 and 2.11, we observe that

$$
\begin{aligned}
\left\|x_{n+1}-z\right\|^{2}= & \left\|\epsilon_{n} \gamma f\left(x_{n}\right)+\beta_{n} x_{n}+\left(\left(1-\beta_{n}\right) I-\epsilon_{n} A\right) k_{n}-q\right\|^{2} \\
\leq & \left\|\left(1-\beta_{n}\right) \frac{\left(\left(1-\beta_{n}\right) I-\epsilon_{n} A\right)}{\left(1-\beta_{n}\right)}\left(k_{n}-q\right)+\beta_{n}\left(x_{n}-q\right)\right\|^{2} \\
& +2 \epsilon_{n}\left\langle\gamma f\left(x_{n}\right)-A q, x_{n+1}-q\right\rangle \\
\leq & \left(1-\beta_{n}\right)\left\|\frac{\left(\left(1-\beta_{n}\right) I-\epsilon_{n} A\right)}{1-\beta_{n}}\left(k_{n}-q\right)\right\|^{2}+\beta_{n}\left\|x_{n}-q\right\|^{2} \\
& +2 \epsilon_{n} \gamma\left\langle f\left(x_{n}\right)-f(q), x_{n+1}-q\right\rangle+2 \epsilon_{n}\left\langle\gamma f(q)-A q, x_{n+1}-q\right\rangle \\
\leq & \left(1-\beta_{n}\right)\left\|\frac{\left(\left(1-\beta_{n}\right) I-\epsilon_{n} A\right)}{1-\beta_{n}}\left(k_{n}-q\right)\right\|^{2}+\beta_{n}\left\|x_{n}-q\right\|^{2} \\
& +2 \epsilon_{n} \gamma \alpha\left\|x_{n}-q\right\|\left\|x_{n+1}-q\right\|+2 \epsilon_{n}\left\langle\gamma f(q)-A q, x_{n+1}-q\right\rangle \\
\leq & \frac{\left\|\left(1-\beta_{n}\right) I-\epsilon_{n} A\right\|^{2}}{1-\beta_{n}}\left\|k_{n}-q\right\|^{2}+\beta_{n}\left\|x_{n}-q\right\|^{2} \\
& +\epsilon_{n} \gamma \alpha\left(\left\|x_{n}-q\right\|^{2}+\left\|x_{n+1}-q\right\|^{2}\right)+2 \epsilon_{n}\left\langle\gamma f(q)-A q, x_{n+1}-q\right\rangle \\
\leq & \frac{\left\|\left(1-\beta_{n}\right) I-\epsilon_{n} A\right\|^{2}}{1-\beta_{n}}\left\|x_{n}-q\right\|^{2}+\beta_{n}\left\|x_{n}-q\right\|^{2} \\
& +\epsilon_{n} \gamma \alpha\left(\left\|x_{n}-q\right\|^{2}+\left\|x_{n+1}-q\right\|^{2}\right)+2 \epsilon_{n}\left\langle\gamma f(q)-A q, x_{n+1}-q\right\rangle
\end{aligned}
$$


Fixed Point Theory and Applications

$$
\begin{aligned}
\leq & \left(\frac{\left(\left(1-\beta_{n}\right)-\bar{\gamma} \epsilon_{n}\right)^{2}}{1-\beta_{n}}+\beta_{n}+\epsilon_{n} \gamma \alpha\right)\left\|x_{n}-q\right\|^{2} \\
& +\epsilon_{n} \gamma \alpha\left\|x_{n+1}-q\right\|^{2}+2 \epsilon_{n}\left\langle\gamma f(q)-A q, x_{n+1}-q\right\rangle \\
\leq & \left(1-(2 \bar{\gamma}-\alpha \gamma) \epsilon_{n}+\frac{\bar{\gamma}^{2} \epsilon_{n}^{2}}{1-\beta_{n}}\right)\left\|x_{n}-q\right\|^{2} \\
& +\epsilon_{n} \gamma \alpha\left\|x_{n+1}-q\right\|^{2}+2 \epsilon_{n}\left\langle\gamma f(q)-A q, x_{n+1}-q\right\rangle
\end{aligned}
$$

which implies that

$$
\begin{aligned}
\left\|x_{n+1}-z\right\|^{2} \leq & \left(1-\frac{2(\bar{\gamma}-\alpha \gamma) \epsilon_{n}}{1-\alpha \gamma \epsilon_{n}}\right)\left\|x_{n}-q\right\|^{2} \\
& +\frac{\epsilon_{n}}{1-\alpha \gamma \epsilon_{n}}\left\{\frac{\bar{\gamma}^{2} \epsilon_{n}^{2}}{1-\beta_{n}}\left\|x_{n}-q\right\|^{2}+2 \epsilon_{n}\left\langle\gamma f(q)-A q, x_{n+1}-q\right\rangle\right\} .
\end{aligned}
$$

Taking

$$
\begin{gathered}
\sigma_{n}=\frac{\epsilon_{n}}{1-\alpha \gamma \epsilon_{n}}\left\{\frac{\bar{\gamma}^{2} \epsilon_{n}^{2}}{1-\beta_{n}}\left\|x_{n}-q\right\|^{2}+2 \epsilon_{n}\left\langle\gamma f(q)-A q, x_{n+1}-q\right\rangle\right\} \\
\rho_{n}=\frac{2(\bar{\gamma}-\alpha \gamma) \epsilon_{n}}{1-\alpha \gamma \epsilon_{n}} .
\end{gathered}
$$

Then we can rewrite (3.75) as

$$
\left\|x_{n+1}-z\right\|^{2} \leq\left(1-\rho_{n}\right)\left\|x_{n}-z\right\|^{2}+\sigma_{n} .
$$

We have $\limsup _{n \rightarrow \infty}\left(\sigma_{n} / \varrho_{n}\right) \leq 0$. Applying Lemma 2.10 to (3.77), we conclude that $\left\{x_{n}\right\}$ converges strongly to $q$ in norm. This completes the proof.

If the mapping $S$ is nonexpansive, then $S_{k}=S_{0}=S$. We can obtain the following result from Theorem 3.1 immediately.

Corollary 3.2. Let $E$ be a nonempty closed convex subset of a real Hilbert space $H$. Let $\phi_{1}$ and $\phi_{2}$ be two bifunction from $E \times E$ to $R$ satisfying $(A 1)-(A 4)$ and let $\varphi: E \rightarrow \mathcal{R} \cup\{+\infty\}$ be a proper lower semicontinuous and convex function. Let $C: E \rightarrow H$ be an $\xi$-inverse strongly monotone mapping and $B: E \rightarrow H$ be an $\beta$-inverse strongly monotone mapping. Let $f: E \rightarrow E$ be a contraction mapping with coefficient $\alpha(0<\alpha<1)$ and let $A$ be a strongly positive linear bounded operator on $H$ with coefficient $\bar{\gamma}>0$ and $0<\gamma<\bar{\gamma} / \alpha$. Let $S: E \rightarrow E$ a nonexpansive mapping with a fixed point. Assume that

$$
\Theta:=F(S) \cap \operatorname{VI}(E, C) \cap \operatorname{VI}(E, B) \cap \operatorname{MEP}\left(\phi_{1}, \varphi\right) \cap \operatorname{MEP}\left(\phi_{2}, \varphi\right) \neq \emptyset .
$$


Assume that either (B1) or (B2). Let $\left\{x_{n}\right\}$ be a sequence generated by the following iterative algorithm:

$$
\begin{gathered}
x_{1} \in E, \quad u_{n} \in E, \quad v_{n} \in E, \\
u_{n}=T_{r}^{\left(\phi_{1}, \varphi\right)} x_{n}, \\
v_{n}=T_{s}^{\left(\phi_{2}, \varphi\right)} x_{n} \\
z_{n}=P_{E}\left(u_{n}-\mu_{n} C u_{n}\right), \\
y_{n}=P_{E}\left(v_{n}-\lambda_{n} B v_{n}\right), \\
k_{n}=a_{n} S x_{n}+b_{n} y_{n}+c_{n} z_{n}, \\
x_{n+1}=\epsilon_{n} \gamma f\left(x_{n}\right)+\beta_{n} x_{n}+\left(\left(1-\beta_{n}\right) I-\epsilon_{n} A\right) k_{n}, \quad \forall n \geq 1,
\end{gathered}
$$

where $\left\{\epsilon_{n}\right\},\left\{\beta_{n}\right\},\left\{a_{n}\right\},\left\{b_{n}\right\}$, and $\left\{c_{n}\right\}$ are sequences in $(0,1)$ and $\left\{\lambda_{n}\right\},\left\{\mu_{n}\right\}$ are positive sequences. Assume that the control sequences satisfy the following restrictions:

(C1) $a_{n}+b_{n}+c_{n}=1$,

(C2) $\lim _{n \rightarrow \infty} \epsilon_{n}=0$ and $\sum_{n=1}^{\infty} \epsilon_{n}=\infty$,

(C3) $0<\liminf _{n \rightarrow \infty} \beta_{n} \leq \lim \sup _{n \rightarrow \infty} \beta_{n}<1$,

(C4) $\lim _{n \rightarrow \infty}\left|\lambda_{n+1}-\lambda_{n}\right|=\lim _{n \rightarrow \infty}\left|\mu_{n+1}-\mu_{n}\right|=0$,

(C5) $d \leq \lambda_{n} \leq 2 \beta, e \leq \mu_{n} \leq 2 \xi$, where $d$, e are two positive constants,

(C6) $\lim _{n \rightarrow \infty} a_{n}=a, \lim _{n \rightarrow \infty} b_{n}=b$ and $\lim _{n \rightarrow \infty} c_{n}=c$, for some $a, b, c \in(0,1)$.

Then, $\left\{x_{n}\right\}$ converges strongly to a point $q \in \Theta$ which is the unique solution of the variational inequality

$$
\langle(A-\gamma f) q, x-q\rangle \geq 0, \quad \forall x \in \Theta
$$

or equivalent $q=P_{\Theta}(I-A+\gamma f)(q)$, where $P$ is a metric projection mapping form $H$ onto $\Theta$.

Finally, we consider the following convex feasibility problem (CFP):

$$
\text { finding an } x \in \bigcap_{i=1}^{N} C_{i}
$$

where $N \geq 1$ is an integer and each $C_{i}$ is assumed to be the of solutions of equilibrium problem with the bifunction $\phi_{i}, i=1,2,3, \ldots, N$ and the solution set of the variational inequality problem. There is a considerable investigation on CEP in the setting of Hilbert spaces which captures applications in various disciplines such as image restoration [42, 43], computer tomography [44], and radiation therapy treatment planning [45].

The following result can be concluded from Theorem 3.1 easily.

Theorem 3.3. Let $E$ be a nonempty closed convex subset of a real Hilbert space $H$. Let be a $\phi_{i}$ bifunction from $E \times E$ to $R$ satisfying $(A 1)-(A 4)$ and let $\varphi: E \rightarrow \mathcal{R} \cup\{+\infty\}$ be a proper lower 
semicontinuous and convex function. Let $C_{i}: E \rightarrow H$ be an $\xi_{\text {inverse strongly monotone mapping }}$ for each $i \in\{1,2,3, \ldots, N\}$. Let $f: E \rightarrow E$ be a contraction mapping with coefficient $\alpha(0<\alpha<1)$ and let $A$ be a strongly positive linear bounded operator on $H$ with coefficient $\bar{\gamma}>0$ and $0<\gamma<\bar{\gamma} / \alpha$. Let $S: E \rightarrow E$ be a $k$-strict pseudo-contraction with a fixed point. Define a mapping $S_{k}: E \rightarrow E$ by $S_{k} x=k x+(1-k) S x$, for all $x \in E$. Assume that

$$
\mathcal{F}:=F(S) \cap\left(\bigcap_{i=1}^{N} \operatorname{VI}\left(E, C_{i}\right)\right) \cap\left(\bigcap_{i=1}^{N} \operatorname{MEP}\left(\phi_{i}, \varphi\right)\right) \neq \emptyset .
$$

Assume that either (B1) or (B2). Let $\left\{x_{n}\right\}$ be a sequence generated by the following iterative algorithm:

$$
\begin{gathered}
x_{1} \in E, \quad u_{n, i} \in E, \\
\phi_{i}\left(u_{n, i}, u_{i}\right)+\varphi\left(u_{i}\right)-\varphi\left(u_{n, i}\right)+\frac{1}{r_{i}}\left\langle u_{i}-u_{n, i}, u_{n, i}-x_{n}\right\rangle \geq 0, \quad \forall u_{i} \in E, \quad \forall i \in\{1,2,3, \ldots, N\}, \\
k_{n}=\alpha_{n, 0} S_{k} x_{n}+\sum_{i=1}^{N} \alpha_{n, i} P_{E}\left(u_{n, i}-\mu_{n, i} C_{i} u_{n, i}\right), \\
x_{n+1}=\epsilon_{n} \gamma f\left(x_{n}\right)+\beta_{n} x_{n}+\left(\left(1-\beta_{n}\right) I-\epsilon_{n} A\right) k_{n}, \quad \forall n \geq 1,
\end{gathered}
$$

where $\alpha_{n, 0}, \alpha_{n, 1}, \alpha_{n, 2}, \alpha_{n, 3}, \ldots, \alpha_{n, N} \in(0,1)$ such that $\sum_{i=0}^{N} \alpha_{n, i}=1,\left\{\mu_{n, i}\right\}$ are positive sequences and $\left\{\epsilon_{n}\right\},\left\{\beta_{n}\right\}$ are sequences in $(0,1)$. Assume that the control sequences satisfy the following restrictions:

(C1) $\lim _{n \rightarrow \infty} \epsilon_{n}=0$ and $\sum_{n=1}^{\infty} \epsilon_{n}=\infty$,

(C2) $0<\liminf _{n \rightarrow \infty} \beta_{n} \leq \lim \sup _{n \rightarrow \infty} \beta_{n}<1$,

(C3) $\lim _{n \rightarrow \infty}\left|\mu_{n+1, i}-\mu_{n, i}\right|=0$, for each $1 \leq i \leq N$,

(C4) $e_{i} \leq \mu_{n, i} \leq 2 \xi_{i}$, where $e_{i}$ is some positive constant for each $1 \leq i \leq N$,

(C5) $\lim _{n \rightarrow \infty} \alpha_{n, i}=\alpha_{i} \in(0,1)$, for each $1 \leq i \leq N$.

Then, $\left\{x_{n}\right\}$ converges strongly to a point $q \in \mathcal{F}$ which is the unique solution of the variational inequality

$$
\langle(A-\gamma f) q, x-q\rangle \geq 0, \quad \forall x \in \mathcal{F}
$$

or equivalent $q=P_{\mathcal{F}}(I-A+\gamma f)(q)$, where $P$ is a metric projection mapping form $H$ onto $\mathcal{F}$.

\section{Acknowledgments}

The authors would like to thank the referees for their valuable suggestions to improve this paper. This work was supported by the Center of Excellence in Mathematics, the Commission on Higher Education, Thailand. 


\section{References}

[1] W. Takahashi, Nonlinear Functional Analysis, Yokohama Publishers, Yokohama, Japan, 2000.

[2] F. E. Browder and W. V. Petryshyn, "Construction of fixed points of nonlinear mappings in Hilbert space," Journal of Mathematical Analysis and Applications, vol. 20, pp. 197-228, 1967.

[3] G. Marino and H.-K. Xu, "Weak and strong convergence theorems for strict pseudo-contractions in Hilbert spaces," Journal of Mathematical Analysis and Applications, vol. 329, no. 1, pp. 336-346, 2007.

[4] H. Zhou, "Convergence theorems of fixed points for $\kappa$-strict pseudo-contractions in Hilbert spaces," Nonlinear Analysis. Theory, Methods E Applications. Series A, vol. 69, no. 2, pp. 456-462, 2008.

[5] L.-C. Ceng and J.-C. Yao, "A hybrid iterative scheme for mixed equilibrium problems and fixed point problems," Journal of Computational and Applied Mathematics, vol. 214, no. 1, pp. 186-201, 2008.

[6] E. Blum and W. Oettli, "From optimization and variational inequalities to equilibrium problems," The Mathematics Student, vol. 63, no. 1-4, pp. 123-145, 1994.

[7] J. C. Yao and O. Chadli, "Pseudomonotone complementarity problems and variational inequalities," in Handbook of Generalized Convexity and Monotonicity, J. P. Crouzeix, N. Haddjissas, and S. Schaible, Eds., pp. 501-558, 2005.

[8] L. C. Zeng, S. Schaible, and J. C. Yao, "Iterative algorithm for generalized set-valued strongly nonlinear mixed variational-like inequalities," Journal of Optimization Theory and Applications, vol. 124, no. 3, pp. 725-738, 2005.

[9] K. Aoyama, Y. Kimura, and W. Takahashi, "Maximal monotone operators and maximal monotone functions for equilibrium problems," Journal of Convex Analysis, vol. 15, no. 2, pp. 395-409, 2008.

[10] P. L. Combettes and S. A. Hirstoaga, "Equilibrium programming using proximal-like algorithms," Mathematical Programming, vol. 78, no. 1, pp. 29-41, 1997.

[11] X. Gao and Y. Guo, "Strong convergence of a modified iterative algorithm for mixed-equilibrium problems in Hilbert spaces," Journal of Inequalities and Applications, vol. 2008, Article ID 454181, 23 pages, 2008.

[12] C. Jaiboon and P. Kumam, "A hybrid extragradient viscosity approximation method for solving equilibrium problems and fixed point problems of infinitely many nonexpansive mappings," Fixed Point Theory and Applications, vol. 2009, Article ID 374815, 32 pages, 2009.

[13] P. Kumam and C. Jaiboon, "A new hybrid iterative method for mixed equilibrium problems and variational inequality problem for relaxed cocoercive mappings with application to optimization problems," Nonlinear Analysis. Hybrid Systems, vol. 3, no. 4, pp. 510-530, 2009.

[14] C. Jaiboon and P. Kumam, "Strong convergence theorems for solving equilibrium problems and fixed point problems of $\xi$-strict pseudo-contraction mappings by two hybrid projection methods," Journal of Computational and Applied Mathematics, vol. 234, no. 3, pp. 722-732, 2010.

[15] C. Jaiboon, P. Kumam, and U. W. Humphries, "Weak convergence theorem by an extragradient method for variational inequality, equilibrium and fixed point problems," Bulletin of the Malaysian Mathematical Sciences Society. Second Series, vol. 32, no. 2, pp. 173-185, 2009.

[16] P. Kumam and C. Jaiboon, "A system of generalized mixed equilibrium problems and fixed point problems for pseudocontractive mappings in Hilbert spaces," Fixed Point Theory and Applications, vol. 2010, Article ID 361512, 33 pages, 2010.

[17] J.-W. Peng and J.-C. Yao, "Strong convergence theorems of iterative scheme based on the extragradient method for mixed equilibrium problems and fixed point problems," Mathematical and Computer Modelling, vol. 49, no. 9-10, pp. 1816-1828, 2009.

[18] X. Qin, Y. J. Cho, and S. M. Kang, "Convergence analysis on hybrid projection algorithms for equilibrium problems and variational inequality problems," Mathematical Modelling and Analysis, vol. 14, no. 3, pp. 335-351, 2009.

[19] S. Takahashi and W. Takahashi, "Viscosity approximation methods for equilibrium problems and fixed point problems in Hilbert spaces," Journal of Mathematical Analysis and Applications, vol. 331, no. 1, pp. 506-515, 2007.

[20] S. Takahashi and W. Takahashi, "Strong convergence theorem for a generalized equilibrium problem and a nonexpansive mapping in a Hilbert space," Nonlinear Analysis. Theory, Methods E Applications, vol. 69, no. 3, pp. 1025-1033, 2008.

[21] W. Takahashi and K. Zembayashi, "Strong convergence theorem by a new hybrid method for equilibrium problems and relatively nonexpansive mappings," Fixed Point Theory and Applications, vol. 2008, Article ID 528476, 11 pages, 2008. 
[22] W. Takahashi and K. Zembayashi, "Strong and weak convergence theorems for equilibrium problems and relatively nonexpansive mappings in Banach spaces," Nonlinear Analysis. Theory, Methods $\mathcal{E}$ Applications, vol. 70, no. 1, pp. 45-57, 2009.

[23] Y. Yao, Y.-C. Liou, and J.-C. Yao, "A new hybrid iterative algorithm for fixed-point problems, variational inequality problems, and mixed equilibrium problems," Fixed Point Theory and Applications, vol. 2007, Article ID 417089, 15 pages, 2008.

[24] Y. Yao, Y.-C. Liou, and Y.-J. Wu, "An extragradient method for mixed equilibrium problems and fixed point problems," Fixed Point Theory and Applications, vol. 2009, Article ID 632819, 15 pages, 2009.

[25] X. Gao and Y. Guo, "Strong convergence of a modified iterative algorithm for mixed-equilibrium problems in Hilbert spaces," Journal of Inequalities and Applications, vol. 2008, Article ID 454181, 23 pages, 2008.

[26] W.-Y. Zeng, N.-J. Huang, and C.-W. Zhao, "Viscosity approximation methods for generalized mixed equilibrium problems and fixed points of a sequence of nonexpansive mappings," Fixed Point Theory and Applications, vol. 2008, Article ID 714939, 15 pages, 2008.

[27] Y. Yao, Y.-C. Liou, and J.-C. Yao, "A new hybrid iterative algorithm for fixed-point problems, variational inequality problems, and mixed equilibrium problems," Fixed Point Theory and Applications, vol. 2008, Article ID 417089, 15 pages, 2008.

[28] R. T. Rockafellar, "On the maximality of sums of nonlinear monotone operators," Transactions of the American Mathematical Society, vol. 149, pp. 75-88, 1970.

[29] W. Takahashi and M. Toyoda, "Weak convergence theorems for nonexpansive mappings and monotone mappings," Journal of Optimization Theory and Applications, vol. 118, no. 2, pp. 417-428, 2003.

[30] Y. Yao and J.-C. Yao, “On modified iterative method for nonexpansive mappings and monotone mappings," Applied Mathematics and Computation, vol. 186, no. 2, pp. 1551-1558, 2007.

[31] G. Marino and H.-K. Xu, "A general iterative method for nonexpansive mappings in Hilbert spaces," Journal of Mathematical Analysis and Applications, vol. 318, no. 1, pp. 43-52, 2006.

[32] S. Plubtieng and R. Punpaeng, "A general iterative method for equilibrium problems and fixed point problems in Hilbert spaces," Journal of Mathematical Analysis and Applications, vol. 336, no. 1, pp. 455469, 2007.

[33] Y. Liu, "A general iterative method for equilibrium problems and strict pseudo-contractions in Hilbert spaces," Nonlinear Analysis. Theory, Methods \& Applications. Series A, vol. 71, no. 10, pp. 4852-4861, 2009.

[34] R. Wangkeeree and R. Wangkeeree, "A general iterative method for variational inequality problems, mixed equilibrium problems, and fixed point problems of strictly pseudocontractive mappings in Hilbert spaces," Fixed Point Theory and Applications, vol. 2009, Article ID 519065, 32 pages, 2009.

[35] X. Qin, Y. J. Cho, and S. M. Kang, "Viscosity approximation methods for generalized equilibrium problems and fixed point problems with applications," Nonlinear Analysis. Theory, Methods $\mathcal{E}$ Applications, vol. 72, no. 1, pp. 99-112, 2010.

[36] M. O. Osilike and D. I. Igbokwe, "Weak and strong convergence theorems for fixed points of pseudocontractions and solutions of monotone type operator equations," Computers $\mathcal{E}$ Mathematics with Applications, vol. 40, no. 4-5, pp. 559-567, 2000.

[37] F. E. Browder, "Nonlinear operators and nonlinear equations of evolution in Banach spaces," Proceedings of Symposia in Pure Mathematics, vol. 18, pp. 78-81, 1976.

[38] R. E. Bruck Jr., "Properties of fixed-point sets of nonexpansive mappings in Banach spaces," Transactions of the American Mathematical Society, vol. 179, pp. 251-262, 1973.

[39] J.-W. Peng and J.-C. Yao, "A new hybrid-extragradient method for generalized mixed equilibrium problems, fixed point problems and variational inequality problems," Taiwanese Journal of Mathematics, vol. 12, no. 6, pp. 1401-1432, 2008.

[40] T. Suzuki, "Strong convergence of Krasnoselskii and Mann's type sequences for one-parameter nonexpansive semigroups without Bochner integrals," Journal of Mathematical Analysis and Applications, vol. 305, no. 1, pp. 227-239, 2005.

[41] H.-K. Xu, "Viscosity approximation methods for nonexpansive mappings," Journal of Mathematical Analysis and Applications, vol. 298, no. 1, pp. 279-291, 2004.

[42] P. L. Combettes, "The convex feasibility problemml: in image recovery," in Advances in Imaging and Electron Physics, P. Hawkes, Ed., vol. 95, pp. 155-270, Academic Press, Orlando, Fla, USA, 1996. 
[43] T. Kotzer, N. Cohen, and J. Shamir, "Images to ration by a novel method of parallel projection onto constraint sets," Optics Letters, vol. 20, pp. 1172-1174, 1995.

[44] M. I. Sezan and H. Stark, "Application of convex projection theory to image recovery in tomograph and related areas," in Image Recovery: Theory and Application, H. Stark, Ed., pp. 155-270, Academic Press, Orlando, Fla, USA, 1987.

[45] Y. Censor and S. A. Zenios, Parallel Optimization, Numerical Mathematics and Scientific Computation, Oxford University Press, New York, NY, USA, 1997. 